\title{
Article
}

\section{Henselianity in the language of rings}

Anscombe, Sylvy and Jahnke, Franziska

Available at http://clok.uclan.ac.uk/22532/

Anscombe, Sylvy ORCID: 0000-0002-9930-2804 and Jahnke, Franziska (2018) Henselianity in the language of rings. Annals of Pure and Applied Logic, 169 (9). pp. 872-895. ISSN 0168-0072

It is advisable to refer to the publisher's version if you intend to cite from the work. http://dx.doi.org/10.1016/j.apal.2018.04.008

For more information about UCLan's research in this area go to http://www.uclan.ac.uk/researchgroups/ and search for < name of research Group>.

For information about Research generally at UCLan please go to http://www.uclan.ac.uk/research/

All outputs in CLoK are protected by Intellectual Property Rights law, including Copyright law. Copyright, IPR and Moral Rights for the works on this site are retained by the individual authors and/or other copyright owners. Terms and conditions for use of this material are defined in the policies page.

\section{CLoK}

Central Lancashire online Knowledge www.clok.uclan.ac.uk

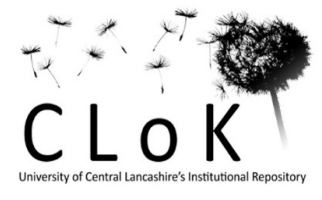




\title{
HENSELIANITY IN THE LANGUAGE OF RINGS
}

\author{
SYLVY ANSCOMBE AND FRANZISKA JAHNKE
}

\begin{abstract}
We consider four properties of a field $K$ related to the existence of (definable) henselian valuations on $K$ and on elementarily equivalent fields and study the implications between them. Surprisingly, the full pictures look very different in equicharacteristic and mixed characteristic.
\end{abstract}

\section{INTRODUCTION}

The study of henselian fields in the language of rings started with a work by Prestel and Ziegler ([PZ78]) where they introduced and discussed $t$-henselian fields. We say that a field is $t$-henselian if it is $\mathcal{L}_{\text {ring-elementarily equivalent to some henselian field, i.e., a }}$ field admitting a nontrivial henselian valuation. Although this does not coincide with the definition given in [PZ78], our definition and theirs are equivalent, using the $\mathcal{L}_{\text {ring- }}{ }^{-}$ definition of the henselian topology in [Pre91, p. 203]. Real closed fields and algebraically closed fields of positive characteristic are t-henselian but may not be henselian, e.g. $\mathbb{R}$ and $\overline{\mathbb{F}}_{p}$ are t-henselian but not henselian. In particular, Prestel and Ziegler showed that these are not the only examples of t-henselian fields which are not henselian. These results are strongly linked to the question of which fields admit a nontrivial definable henselian valuation. Here, we say that a valuation $v$ is definable on a field $K$ if its valuation

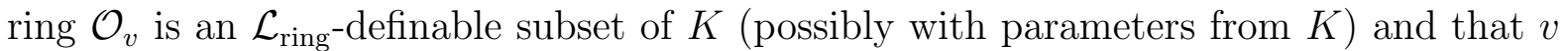
is $\emptyset$-definable if it is definable and no parameters were needed in the defining formula. Henselianity is an elementary property of valued fields, in particular, it is preserved under elementary equivalence in the language $\mathcal{L}_{\text {val }}=\mathcal{L}_{\text {ring }} \cup\{\mathcal{O}\}$ where the unary relation symbol $\mathcal{O}$ is interpreted as the valuation ring. Thus, if some nontrivial henselian valuation ring is a $\emptyset$-definable subring of $K$, then any $L$ which is $\mathcal{L}_{\text {ring }}$-elementarily equivalent to $K$ also admits a nontrivial henselian valuation. In particular, if $K$ is henselian and some $\mathcal{L}_{\text {ring- }}$ elementarily equivalent $L$ is non-henselian, then $K$ cannot admit a $\emptyset$-definable nontrivial henselian valuation. Under which conditions fields admit definable nontrivial henselian valuations (with or without parameters) has been investigated in a number of (mostly) recent papers ([Hon14], JK15a, JK15b, KKoe94, Pre15]) and some of these results have been applied in connection with the Shelah-Hasson conjecture on NIP fields (see [Joh15] and [Kru15]).

The aim of this paper is to clarify the implications and relationships between these properties of a field $K$, more precisely:

(h) $K$ is henselian (i.e., $K$ admits a nontrivial henselian valuation),

(eh) any $L$ which is $\mathcal{L}_{\text {ring }}$-elementarily equivalent to $K$ is henselian,

( -def) $K$ admits a $\emptyset$-definable nontrivial henselian valuation, and

(def) $K$ admits a definable nontrivial henselian valuation.

April 25, 2018

This work was begun while the first author was funded by EPSRC grant EP/K020692/1, the second author received funding from SFB 878. 
We call a field elementarily henselian if it satisfies (eh). There are some immediate implications between these properties, as summarised in the following diagram: 1

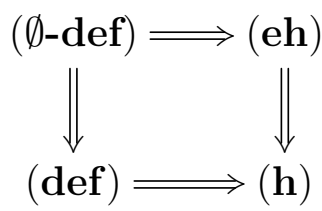

Figure 1: The obvious implications

Our aim is to work out the full picture, i.e., to describe which other implications hold, including which arrows can be reversed. It turns out that in the class of all fields (or even in the class $\mathcal{K}_{0}$ of all non-algebraically closed fields of characteristic zero), no implications hold that are not already included in Figure 1 (see part (C) of Theorem 1.1).

In order to show this, we use the canonical henselian valuation $v_{K}$ to partition $\mathcal{K}_{0}$ into subclasses, depending on the residue characteristic of $v_{K}$ :

$$
\mathcal{K}_{0,0}=\left\{K \text { field } \mid \operatorname{char}(K)=\operatorname{char}\left(K v_{K}\right)=0, K \text { not algebraically closed }\right\}
$$

and for any prime $p$

$$
\mathcal{K}_{0, p}=\left\{K \text { field } \mid \operatorname{char}(K)=0 \text { and } \operatorname{char}\left(K v_{K}\right)=p\right\} .
$$

See section 2 for the definition of the canonical henselian valuation and a proof that

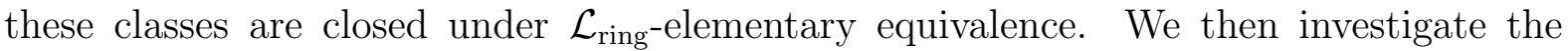
corresponding pictures with respect to these subclasses which surprisingly turn out to look rather different in mixed characteristic and equicharacteristic 0. As our main result, we obtain the following

Theorem 1.1. (A) In the class $\mathcal{K}_{0,0}$ the complete picture is

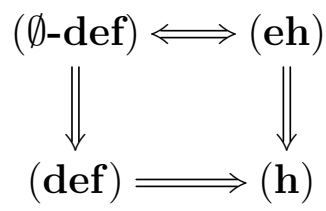

(B) For each prime $p$, in the class $\mathcal{K}_{0, p}$ the complete picture is

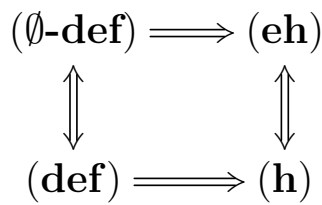

(C) Consequently, in the class $\mathcal{K}_{0}$ the complete picture is given by Figure 1 .

On an algebraically closed field, any valuation is henselian. Thus, any algebraically closed fields of characteristic zero admits many nontrivial henselian valuations (for example, consider any extension of the $p$-adic valuation, for a prime $p$ ). Since any algebraically closed field is strongly minimal, every definable subset is either finite or cofinite. In particular, no such field can admit a definable nontrivial henselian valuation. Therefore,

\footnotetext{
${ }^{1}$ Our convention is that such diagrams implicitly include concatenations of arrows, although we do not draw them. For example, Figure 1 implicitly includes the implication $(\emptyset$-def $) \Longrightarrow(\mathbf{h})$.
} 
algebraically closed fields of characteristic zero satisfy both (h) and (eh), and neither (def) nor $(\emptyset$-def $)$.

The paper is organized as follows. In the next subsection (subsection 1.1), we introduce the basic terminology which we use throughout the paper and discuss the implications and non-implications in our diagrams which are already known.

In section 2, we recall the definition of the canonical henselian valuation $v_{K}$ and show that certain properties of the valued field $\left(K, v_{K}\right)$ are preserved under elementary equivalence in $\mathcal{L}_{\text {ring }}$ (Proposition 2.1). In particular, we obtain that the classes $\mathcal{K}_{0,0}$ and $\mathcal{K}_{0, p}$ (for a fixed prime $p$ ) are closed under $\mathcal{L}_{\text {ring }}$-elementary equivalence.

In section 3, we show part (A) of Theorem 1.1. In order to do this, we first show the implication which occurs in the picture in (A) but not in Figure 1 (see Proposition 3.5). We then combine this with the examples discussed in subsection 1.1 to complete the proof of Theorem 1.1 (A) (see subsection 3.2).

The proof of part (B) of Theorem 1.1 takes some more work. Section 4 treats the constructions which we use to show the non-implications in the diagram: The main result of this section is the existence of non-henselian $t$-henselian fields $K$ (which are neither real closed nor separably closed) of any characteristic such that there is some tame $L \equiv K$ with divisible value group (see subsection 4.2 for the definition of 'tame', and Proposition 4.13 for the statement).

In subsection 5.2, we use the fields constructed in section 4 and the machinery developed in subsection 5.1 to show that for every prime $p$, there are fields in $\mathcal{K}_{0, p}$ which do not admit $\emptyset$-definable nontrivial henselian valuations (see Example 5.5). We then go on to show that for every prime $p$ and every $K \in \mathcal{K}_{0, p}$, the properties (def) and $(\emptyset$-def) are equivalent (see Theorem 5.7). Finally, we assemble the facts we have shown about fields in $\mathcal{K}_{0, p}$ to prove Theorem 1.1 (B) in subsection 5.4.

1.1. Preliminaries and known results. For basic definitions and notions regarding valuation theory, we refer the reader to EP05. We use the following notation: If $(K, v)$ is a valued field, we let $\mathcal{O}_{v}$ denote the valuation ring, $\mathfrak{m}_{v}$ denote the maximal ideal, $K v$ denote the residue field, and $v K$ denote the value group. For $a \in \mathcal{O}_{v}$, let $\bar{a}$ denote the residue of $a$.

The properties $(\mathbf{e h})$ and $\left(\emptyset\right.$-def) are obviously preserved under $\mathcal{L}_{\text {ring }}$-elementary equivalence. Some real closed fields are henselian, for example $\mathbb{R}\left(\left(x^{\mathbb{Q}}\right)\right)$; and others are not, for example $\mathbb{R}$. Here $\mathbb{R}\left(\left(x^{\mathbb{Q}}\right)\right)$ denotes the field of generalized power series with coefficients from $\mathbb{R}$ and exponents in $\mathbb{Q}$, see [Efr06, 4.2]. This field admits a unique nontrivial henselian valuation $v_{x}$, namely the power series valuation with residue field $\mathbb{R}$ and value group $\mathbb{Q}$. In fact, $v_{x}$ is the canonical henselian valuation on this field, see section 2 for details. The completeness of the theory of real closed fields shows that

(1) $(\mathbf{h})$ is not preserved under $\mathcal{L}_{\text {ring-elementary equivalence and }}$

(2) (h) does not imply (eh) for fields in $\mathcal{K}_{0,0}$.

Since real closed fields are o-minimal, no real closed field admits a definable nontrivial henselian valuation. Thus

(3) (h) does not imply (def) for fields in $\mathcal{K}_{0,0}$.

Consequently, (h) implies neither $(\mathbf{e h})$ nor $(\mathbf{d e f})$, for all fields in $\mathcal{K}_{0}$. However, even if we exclude real closed fields, these implications do not hold: for (2) this is shown by an example of Prestel and Ziegler in [PZ78, p. 338], and for (3) this is shown by an example of Jahnke and Koenigsmann in [JK15a, Example 6.2].

Furthermore, Jahnke and Koenigsmann give an example of a henselian field which does admit a nontrivial definable henselian valuation but does not admit a nontrivial 
$\emptyset$-definable henselian valuation ([JK15a, Example 6.3]). In fact, the field $K$ constructed

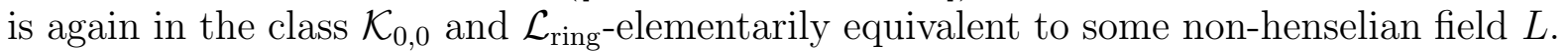
Thus, we get

(4) (def) is not preserved under $\mathcal{L}_{\text {ring-elementary equivalence, }}$

(5) (def) does not imply $\left(\emptyset\right.$-def) in $\mathcal{K}_{0,0}$ (and hence in $\mathcal{K}_{0}$ ).

However, even in the equicharacteristic zero setting there are unanswered questions. Perhaps the most obvious is the following, which is labelled 'Question 5.6' in [JK15a.

Question 1.2. Does (eh) imply ( $\emptyset$-def) for non-separably closed fields?

We answer this question negatively for the class $\mathcal{K}_{0}$, however, we show that it does hold when we restrict our attention to $\mathcal{K}_{0,0}$ (see Proposition 3.5.

\section{THE CANONICAL HENSELIAN VALUATION}

Recall that any henselian field $K$ may admit many non-trivial henselian valuations. However, unless $K$ is separably closed, these all induce the same topology on $K$. This fact ensures that there is always a canonical one among the henselian valuations on a field. The canonical henselian valuation $v_{K}$ on $K$ is defined as follows: We divide the class of henselian valuations on $K$ into subclasses, namely

$$
H_{1}(K)=\{v \text { henselian on } K \mid K v \text { not separably closed }\}
$$

and

$$
H_{2}(K)=\{v \text { henselian on } K \mid K v \text { separably closed }\}
$$

If $H_{2}(K) \neq \emptyset$, i.e., if $K$ admits a henselian valuation with separably closed residue field, then $v_{K}$ is the (unique) coarsest such. In particular, we have $v_{K} \in H_{2}(K)$. In this case, any henselian valuation with non-separably closed residue field is a proper coarsening of $v_{K}$ and any henselian valuation with separably closed residue field is a refinement of $v_{K}$.

If $H_{2}(K)=\emptyset$, i.e., if $K$ admits no henselian valuations with separably closed residue field, then $v_{K}$ is the (unique) finest henselian valuation on $K$ and any two henselian valuations on $K$ are comparable. In this case, we have $v_{K} \in H_{1}(K)$.

In any case, we denote by $\mathcal{O}_{K}$ the valuation ring of $v_{K}$. Note that whenever $K$ is not separably closed and admits some nontrivial henselian valuation then $v_{K}$ is nontrivial, i.e., we have $\mathcal{O}_{K} \subsetneq K$. In case $K$ is separably closed, we let $v_{K} \in H_{2}(K)$ be the trivial valuation. See [EP05, §4.4] for more details and proofs.

We now show that certain key properties of the canonical henselian valuation $v_{K}$ on $K$ are preserved under $\mathcal{L}_{\text {ring-elementary equivalence. }}$

Proposition 2.1. Let $p$ be any prime. The following properties of a field $K$ are preserved under $\mathcal{L}_{\text {ring-elementary equivalence: }}$

(1) ' $v_{K} \in H_{2}(K)$ ',

(2) ' $v_{K}$ has residue characteristic $p$ ',

(3) ' $v_{K}$ has residue characteristic zero', and

(4) ' $K$ admits a henselian valuation of mixed characteristic $(0, p)$ '.

Proof. Let $L \equiv K$ be a pair of elementarily equivalent fields. In each case we suppose that the relevant property holds in $K$ and show that it also holds in $L$.

(1) Assume that $v_{K} \in H_{2}(K)$. By compactness, there exists an elementary extension $\left(K, v_{K}\right) \preceq\left(K^{*}, v_{K}^{*}\right)$ such that $L$ elementarily embeds into $K^{*}$; we identify $L$ with its image under this elementary embedding. Let $w$ denote the restriction of $v_{K}^{*}$ to $L$. Since $L$ is relatively algebraically closed in $K^{*},(L, w)$ is henselian. By Hensel's 
Lemma, $L w$ is relatively separably algebraically closed in $K^{*} v_{K}^{*}$, and the latter is separably closed. Thus $L w$ is separably closed. Therefore we get $w \in H_{2}(L)$ and hence $H_{2}(L) \neq \emptyset$. We conclude $v_{L} \in H_{2}(L)$.

Both parts (2) and (3) follow from the following claim.

Claim 2.1.1. If $K \equiv L$, then the residue characteristics of $v_{K}$ and $v_{L}$ are equal.

Proof of claim. We will distinguish two cases, based on whether or not $H_{2}(K)$ is empty. By part $(1), H_{2}(K)$ is empty if and only if $H_{2}(L)$ is empty. In each case we will use again the construction from part (1) in which we identify $L$ with an elementary subfield of $K^{*}$, where $\left(K^{*}, v_{K}^{*}\right)$ is an elementary extension of $\left(K, v_{K}\right)$. We let $w$ denote the restriction of $v_{K}^{*}$ to $L$. Since $L$ is relatively algebraically closed in $K^{*}, w$ is henselian; and thus $L w$ is relatively separably closed in $K^{*} v_{K}^{*}$.

(i) First we suppose that $H_{2}(K)=\emptyset$. It suffices to show that if one of $\left(K, v_{K}\right)$ and $\left(L, v_{L}\right)$ has residue characteristic $p$, then so has the other. Without loss of generality, we suppose that $\operatorname{char}\left(K v_{K}\right)=p$. Then $\operatorname{char}\left(K^{*} v_{K}^{*}\right)=p$; and since $w$ is a restriction of $v_{K}^{*}$, we have that $\operatorname{char}(L w)=p$. As $H_{2}(L)=\emptyset$ holds, $v_{L}$ is a (possibly improper) refinement of $w$. Thus $\operatorname{char}\left(L v_{L}\right)=p$, as required.

(ii) Next we suppose that $H_{2}(K) \neq \emptyset$. We first show that if one of $\left(K, v_{K}\right)$ and $\left(L, v_{L}\right)$ has residue characteristic zero, then so has the other. Without loss of generality, we suppose that $\operatorname{char}\left(K v_{K}\right)=0$. Then $\operatorname{char}(L w)=0$. Since $v_{K} \in H_{2}(K), K v_{K}$ and $K^{*} v_{K}^{*}$ are separably closed fields. Since $L w$ is relatively separably closed in $K^{*} v_{K}^{*}, L w$ is also separably closed. Thus $w$ is a (possibly improper) refinement of $v_{L}$. Thus $\operatorname{char}\left(L v_{L}\right)=0$, as required.

Now, assume char $\left(K v_{K}\right)=p>0$. In particular, for any $w$ henselian on $K$ we have $\operatorname{char}(K w) \in\{0, p\}$. Take any elementary extension $M$ of $K$. Then, we have $\operatorname{char}\left(M v_{M}\right)>0$ by the above, and the restriction of $v_{M}$ to $K$ is a henselian valuation of mixed characteristic. We conclude $\operatorname{char}\left(K v_{K}\right)=\operatorname{char}\left(M v_{M}\right)$. For any $L \equiv K$ there is some $M$ such that both $K$ and $L$ embed elementarily into $M$. Thus, we get $\operatorname{char}\left(K v_{K}\right)=\operatorname{char}\left(L v_{L}\right)$.

This completes the proof of the claim.

(4) Suppose that $K$ admits a henselian valuation $v$ of mixed characteristic $(0, p)$, for a prime $p$. If $v_{K}$ is of mixed characteristic $(0, p)$ then we simply apply part $(2)$. Otherwise $v_{K}$ is of residue characteristic zero and $v$ is a proper refinement of $v_{K}$. Thus $v_{K} \in H_{2}(K)$, and both $K v_{K}$ and $K v$ are separably closed fields. By parts (1) and (3), $L v_{L}$ is also a separably closed field of characteristic zero. Such fields always carry nontrivial henselian valuations of mixed-characteristic $(0, p)$. Since the composition of two henselian valuations is henselian (see EP05, Corollary 4.1.4]), the composition of $v_{L}$ with any of these gives a nontrivial henselian valuation of mixed-characteristic $(0, p)$ on $L$.

Corollary 2.2. Let $K$ be a field. The property

(mc) ' $K$ admits some mixed characteristic henselian valuation' implies that $K$ is elementarily henselian.

Proof. Assume that $K$ admits a mixed characteristic henselian valuation. By part (4) of Proposition 2.1, all fields $L$ elementarily equivalent to $K$ admit mixed characteristic henselian valuations. Such valuations are necessarily nontrivial. Thus $L$ is henselian.

As a consequence we obtain the following. 
Corollary 2.3. If $K$ is a non-separably closed non-elementarily henselian field then all henselian valuations on fields $L \equiv K$ are equicharacteristic and $H_{2}(L)=\emptyset$.

Proof. Let $L \equiv K$. If $K$ is a non-separably closed non-elementarily henselian field, then so is $L$. By the contrapositive of Corollary 2.2, any henselian valuation on $L$ is equicharacteristic.

Finally, if $H_{2}(L) \neq \emptyset$, then $v_{L} \in H_{2}(L)$. By Proposition 2.1 part 1, we get $v_{M} \in H_{2}(M)$, for any $M \equiv L$. In particular, $L$ is elementarily henselian.

\section{Fields of EQUiCharaCteristic Zero}

In this section, we show part (A) of Theorem 1.1. Note that we only need to show one further arrow to complete the picture, namely (eh) $\Longrightarrow(\emptyset$-def). This is done in subsection 3.1. Afterwards, in subsection 3.2, we explain why combined with the results in subsection 1.1, this indeed proves Theorem 1.1 part (A).

3.1. 'Elementarily henselian' implies ' $\emptyset$-definable'. In this subsection, we show why in the class $\mathcal{K}_{0,0}$ of non-algebraically closed fields $K$ with $\operatorname{char}\left(K v_{K}\right)=0$, the implication $(\mathbf{e h}) \Longrightarrow(\emptyset$-def $)$ holds. We will apply the following theorem from [JK15a].

Theorem 3.1. [JK15a, Theorem B (version given in section 6)] Let $K$ be a non-separably closed henselian field. Then $K$ admits a definable nontrivial henselian valuation (using at most 1 parameter) unless

(1) $K v_{K} \neq K v_{K}^{\mathrm{sep}}$, and

(2) $K v_{K} \preceq L$ for some henselian $L$ with $v_{L} L$ divisible, and

(3) $v_{K} K$ is divisible.

Lemma 3.2. If $K \in \mathcal{K}_{0,0}$ is elementarily henselian then $K$ admits a nontrivial henselian valuation which is definable using at most 1 parameter. In particular, for non-algebraically closed fields of equicharacteristic zero, (eh) implies (def).

Proof. We show the contrapositive. Let $K \in \mathcal{K}_{0,0}$ and suppose that $K$ does not admit a nontrivial henselian valuation which is definable using at most 1 parameter. If $K$ is not henselian then we are done; otherwise $K$ is henselian and we may apply Theorem 3.1. since $K$ is not separably closed. Therefore:

(1) $K v_{K} \neq K v_{K}^{\text {sep }}$, and

(2) $K v_{K} \preceq L$ for some henselian $L$ with $v_{L} L$ divisible, and

(3) $v_{K} K$ is divisible.

Both $\left(K, v_{K}\right)$ and $\left(L, v_{L}\right)$ are henselian valued fields with divisible value groups. By the definition of the canonical henselian valuation, $K v_{K}$ is either separably closed or not henselian. By (1), $K v_{K}$ is not separably closed; thus $K v_{K}$ is not henselian and $L$ is nonelementarily henselian. Applying Corollary 2.3 to $L$, we get that $\operatorname{char}\left(L v_{L}\right)=0$ holds. By applying the Ax-Kochen/Ersov principle ([PD11, Theorem 4.6.4]) several times, we conclude:

$$
\begin{aligned}
K & \equiv K v_{K}\left(\left(x^{\mathbb{Q}}\right)\right) \\
& \equiv L\left(\left(x^{\mathbb{Q}}\right)\right) \\
& \equiv L v_{L}\left(\left(y^{\mathbb{Q}}\right)\right)\left(\left(x^{\mathbb{Q}}\right)\right) \\
& \equiv L .
\end{aligned}
$$

where $\equiv$ is always meant as elementary equivalence in $\mathcal{L}_{\text {ring }}$. Therefore, $K \equiv L \equiv K v_{K}$ holds. Thus, $K$ is not elementarily henselian. 
Definition 3.3. We say that a valuation ring $\mathcal{O}$ on a field $K$ is $n_{\leq}$-henselian if for any separable monic polynomial $f \in \mathcal{O}[X]$ of degree $\leq n$, and any $a \in \mathcal{O}$ with $\bar{f}(\bar{a})=0$ and $\bar{f}^{\prime}(\bar{a}) \neq 0$, there exists an $\alpha \in \mathcal{O}$ with $f(\alpha)=0$ and $\bar{\alpha}=\bar{a}$.

We now want to use Lemma 3.2 to show our missing arrow. The argument works via the Omitting Types Theorem. Thus, we first start by giving names to the relevant (partial) types.

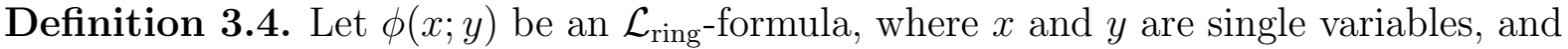
let $n \in \mathbb{N}$. Let $\delta_{\phi, n}(y)$ be the $\mathcal{L}_{\text {ring }}$-formula that defines the set of elements $b$ such that $\phi(x ; b)$ defines a nontrivial $n_{\leq}$-henselian valuation ring. We let $D_{\phi}(y)$ denote the partial type

$$
\left\{\delta_{\phi, n}(y) \mid n<\omega\right\} .
$$

Note that $D_{\phi}(y)$ is realised in $K$ if and only if there exists some $b \in K$ such that $\phi(K ; b)$ is a nontrivial henselian valuation ring of $K$.

Proposition 3.5. If $K \in \mathcal{K}_{0,0}$ is elementarily henselian then $K$ admits a nontrivial $\emptyset$ definable henselian valuation. Equivalently, for non-algebraically closed fields of equicharacteristic zero, we have

$$
(\mathbf{e h}) \Longrightarrow(\emptyset \text {-def }) \text {. }
$$

Proof. First we show that there is a single formula which defines (with parameters) a nontrivial henselian valuation ring in every $L \equiv K$.

Consider the following countable set of partial types (with respect to the theory of $K$ ):

$$
\mathcal{D}:=\left\{D_{\phi}(y) \mid \phi \in \mathcal{L}_{\text {ring }}, D_{\phi}(y) \text { is consistent with } \operatorname{Th}(K)\right\} .
$$

We suppose, seeking a contradiction, that none of these types is principal. By the Omitting Types Theorem (see [TZ12, Corollary 4.1.3]), there exists some $L \equiv K$ in which none of these types is realised. That is: $L$ does not admit a nontrivial definable henselian valuation, defined using at most 1 parameter. Now Lemma 3.2 implies that $L$ is not elementarily henselian, which contradicts our assumption that $K \equiv L$ is elementarily henselian.

Thus there exists an $\mathcal{L}_{\text {ring-formula }} \phi(x ; y)$ such that $D_{\phi}(y)$ is principal. Let $\psi(y)$ be a formula which is consistent and isolates $D_{\phi}(y)$, i.e.

$$
K \models \forall y\left(\psi(y) \longrightarrow \delta_{\phi, n}(y)\right),
$$

for all $n<\omega$. Then $\psi(y)$ defines a nonempty set of realisations of $D_{\phi}(y)$ in any $L \equiv$ $K$. Each element $a$ in this definable set, together with the formula $\phi(x, y)$, defines a nontrivial henselian valuation; that is, we have a $\emptyset$-definable family of nontrivial henselian valuations. It remains to show that we can $\emptyset$-define one such.

If $H_{2}(K) \neq \emptyset$ then there exists a nontrivial $\emptyset$-definable henselian valuation, by [JK15a, Theorem A]. On the other hand, if $H_{2}(K)=\emptyset$, then all henselian valuations on $K$ are pairwise comparable. Let $\Phi(x)$ be the formula

$$
\forall y(\psi(y) \longrightarrow \phi(x ; y)) \text {. }
$$

This formula $\emptyset$-defines the intersection of the $\emptyset$-definable chain of nontrivial henselian valuation rings shown to exist above; and this intersection is also a nontrivial henselian valuation ring. 
3.2. The full picture in equicharacteristic zero. We are now in a position to give the following:

Proof of part (A) of Theorem 1.1. Our aim is to establish that the complete picture of implications in the class $\mathcal{K}_{0,0}$ is given by the following diagram.

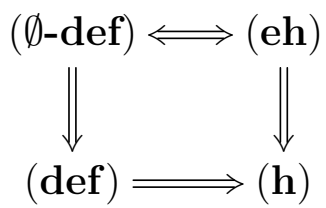

The implication $(\mathbf{e h}) \Longrightarrow(\emptyset$-def $)$ was shown in Proposition 3.5. The other implications in the above diagram already hold in the class of all fields (see Figure 1). Finally, the discussion in subsection 1.1 shows that any implication not contained in the above diagram does not hold in the class $\mathcal{K}_{0,0}$.

\section{FieldS OF DIVISIBLE-TAME TYPE}

The aim of this section is to construct a t-henselian but non-henselian field of divisibletame type (see Definition 4.4), in any given characteristic. Later, specifically in Example 5.5, we will rely on the existence of such fields.

Our construction is a slight modification of that found in the recent paper [FJ15]. It has three main stages. In Lemma 4.8 we construct a valuation with prescribed residue field and satisfying various properties. Then in Lemma 4.11 we study 'infinite compositions' of such valuations. In the final step, which is Proposition 4.13, we combine these lemmas to construct the t-henselian and non-henselian field of divisible-tame type, as required.

4.1. Defectless and $n_{<}$-defectless valued fields. We begin by defining and studying ' $n_{\leq}$-defectless' valued fields. This notion is a weakening of the usual notion of 'defectless', and it is central to our construction.

Definition 4.1. A valued field $(K, v)$ is $n_{\leq}$-defectless if the fundamental equality

$$
[L: K]=[L w: K v] \cdot(w L: v K)
$$

holds for each extension $(L, w) /(K, v)$ of degree $\leq n$. A valued field $(K, v)$ is defectless if it is $n_{\leq}$-defectless for all $n \in \mathbb{N}$.

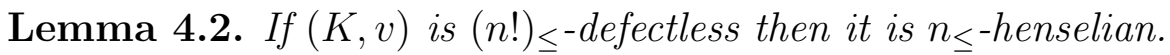

Proof. By the fundamental inequality (see [EP05, Theorem 3.3.4]), $v$ must extend uniquely to every Galois extension of degree $\leq n$ !. By [FJ15, Lemma 6.3(2)], it follows that $(K, v)$ is $n_{\leq}$-henselian.

4.2. 'Divisible-tame' type, and $q$-henselianity. We recall the following definition.

Definition 4.3. A valued field $(K, v)$ of residue characteristic $p$ is tame if $v$ is henselian, the residue field $K v$ is perfect, the value group $v K$ is $p$-divisible, and $(K, v)$ is defectless.

For more detail on tame valued fields we refer the reader to [Kuh16].

Definition 4.4. We say that a t-henselian field $k$ is of divisible-tame type if there exists some $K \equiv k$ and a nontrivial valuation $v$ on $K$ such that $(K, v)$ is tame and $v K$ is divisible.

One variant of henselianity is $n_{\leq}$-henselianity, as defined in Definition 3.3. Another is ' $q$-henselianity', which we recall in the next definition. 
Definition 4.5. Let $(K, v)$ be a valued field and $q$ a prime. We say that $(K, v)$ is $q$-henselian if $v$ extends uniquely to every Galois extension of $K$ of $q$-power degree.

See [FJ15] for some further details on $n_{\leq}$-henselian valuations and [JK15b] for more on $q$-henselian valuations. We will encounter $q$-henselian valuations again in subsection 5.3. Note that if $(K, v)$ is henselian, then it is $n_{\leq}$-henselian for all $n \in \mathbb{N}$ and $q$-henselian for all primes $q$.

4.3. The construction, stage I. Now we come to the construction, which aims to build a t-henselian field of divisible-tame type which is not henselian. We begin by recalling the following fact, which is sometimes known as 'Galois' Translation Theorem'. For a field $K$, we denote its absolute Galois group by $G_{K}$.

Fact 4.6 (cf. [Lan05, Theorem 4.5]). Let $K / F$ be a Galois extension with group G. Let $E$ be an arbitrary extension field of $F$. Assume that $K, E$ are both contained in some field, and let $K E$ be the composite field. Then KE is Galois over E. The map

$$
G_{K E / E} \longrightarrow G_{K / F} \text { given by } \sigma \longmapsto \operatorname{res}_{K}(\sigma) \text {, }
$$

i.e. the restriction of an element of $G_{K E / E}$ to $K$, gives an isomorphism of $G_{K E / E}$ with $G_{K /(K \cap E)}$.

The following lemma, from [FJ15], is a key step in the construction in that paper. Let $\mathbb{P}$ be the set of prime numbers.

Lemma 4.7 ([FJ15, Lemma 6.4]). Let $K_{0}$ be a field of characteristic zero that contains all roots of unity. Let $n \in \mathbb{N}, n<q \in \mathbb{P}$, and $P \subseteq \mathbb{P}$. Then there exists a valued field $\left(K_{1}, v\right)$ with the following properties:

(1) $K_{1} v=K_{0}$ and $v K_{1}=\mathbb{Z}\left[\frac{1}{p}: p \in \mathbb{P} \backslash P\right]$

(2) $v$ is $n_{\leq}$-henselian but not q-henselian

(3) $G_{K_{1}}=\left\langle H_{1}, H_{2}\right\rangle$, where $H_{1} \cong \mathbb{Z}_{q}$ and there is $N \triangleleft H_{2}$ closed with $N \cong \prod_{p \in P} \mathbb{Z}_{p}$ and $H_{2} / N \cong G_{K_{0}}$.

The first stage of our construction, Lemma 4.8, is to give a new version of this lemma which is suitable for arbitrary characteristic. Although parts of our argument vary only slightly from the proof of [FJ15, Lemma 6.4], we nevertheless give the proof in full for the convenience of the reader.

Lemma 4.8. Let $p$ be a prime or zero and let $K$ be a perfect field of characteristic $p$ that contains all roots of unity. Let $n \in \mathbb{N}$ with $p<n$, and let $q$ be a prime such that $\left(n !^{2} !\right)<q$. Then there exists an equicharacteristic valued field $\left(K^{\prime}, v\right)$ such that

(1) $K^{\prime} v=K$

(2) $v K^{\prime}=\mathbb{Q}$,

(3) $K^{\prime}$ is perfect,

(4) $\left(K^{\prime}, v\right)$ is not q-henselian,

(5) $\left(K^{\prime}, v\right)$ is $\left(n !^{2} !\right)_{\leq}$-henselian, and

(6) $\left(K^{\prime}, v\right)$ is $n_{\leq- \text {defectless. }}$

Proof. We work inside the field $K\left(\left(x^{\mathbb{Q}}\right)\right)$ of generalized power series with exponents in $\mathbb{Q}$, together with the $x$-adic valuation, which we denote by $v_{x}$. In fact, $v_{x}$ will also denote the restriction of the $x$-adic valuation to any subfield of $K\left(\left(x^{\mathbb{Q}}\right)\right)$. Let $F:=K\left(x^{\mathbb{Q}}\right)=$ $K\left(x^{\gamma} \mid \gamma \in \mathbb{Q}\right)$. The valued field $\left(F, v_{x}\right)$ has residue field $F v_{x}=K$ and value group $v_{x} F=\mathbb{Q}$. 
By [AF16, Proposition 4.6], the valued field $\left(K\left(\left(x^{\mathbb{Q}}\right)\right), v_{x}\right)$ is tame. Let $F^{\mathrm{ra}}:=F^{\mathrm{alg}} \cap$ $K\left(\left(x^{\mathbb{Q}}\right)\right)$ denote the relative algebraic closure of $F$ in $K\left(\left(x^{\mathbb{Q}}\right)\right)$ and consider the extension

$$
\left(F^{\mathrm{ra}}, v_{x}\right) \subseteq\left(K\left(\left(x^{\mathbb{Q}}\right)\right), v_{x}\right) .
$$

The corresponding extension of residue fields is trivial, since both residue fields are equal to $K$. In particular the extension of the residue field is algebraic. Thus we may apply [Kuh16, Lemma 3.7] to find that $\left(F^{\mathrm{ra}}, v_{x}\right)$ is tame.

Exactly as in the proof of [FJ15, Lemma 6.4], we argue that there exists a subgroup $G_{q} \leq G_{F}$ with $G_{q} \cong \mathbb{Z}_{q}$, as follows. Let $f$ be the polynomial $T^{q}-(x+1) \in K(x)[T]$. Since $K$ contains $q$-th roots of unity and $p \neq q$, by Hensel's Lemma there is a root $\alpha \in F^{\text {ra }}$ of $f$, and the Galois group of $F(\alpha) / F$ is isomorphic to $C_{q}$, the cyclic group of order $q$. If $Q$ denotes a $q$-Sylow subgroup of $G_{F}$, then the image of $Q$ under the restriction map res : $G_{F} \longrightarrow \operatorname{Gal}(F(\alpha) / F)$ is the entirety of $\operatorname{Gal}(F(\alpha) / F)$. We choose $\sigma \in Q$ such that $\operatorname{res}(\sigma)$ generates $\operatorname{Gal}(F(\alpha) / F)$, and let $G_{q}$ denote the procyclic subgroup of $G_{F}$ generated by $\sigma$. Since $G_{q}$ is procyclic, pro- $q$, and torsion-free, we have that $G_{q} \cong \mathbb{Z}_{q}$.

Let $E$ denote the fixed field of $G_{q}$ and let $K^{\prime}$ be the intersection $E \cap F^{\text {ra }}$. The valued field $\left(K^{\prime}, v_{x}\right)$ has residue field $K^{\prime} v_{x}=K$ and value group $v_{x} K^{\prime}=\mathbb{Q}$. Note also that $K^{\prime}$ is perfect, since it is the intersection of two perfect fields.

It remains to show that $\left(K^{\prime}, v_{x}\right)$ is not $q$-henselian, is $\left(n !^{2} !\right)_{\leq-}$-henselian, and is $n_{\leq^{-}}$ defectless.

Claim 4.8.1. $\left(K^{\prime}, v_{x}\right)$ is not q-henselian.

Proof of claim. Assume that $\left(K^{\prime}, v_{x}\right)$ is $q$-henselian. Note that the splitting field of $f$ over $K^{\prime}$ is a Galois extension of degree $q$. By the $q$-henselian version of Hensel's Lemma (see [EP05, Theorem 4.2.3(2)], $f$ has a root in $K^{\prime}$. This is a contradiction because $f$ has no roots in $E$.

Claim 4.8.2. $\left(K^{\prime}, v_{x}\right)$ is $\left(n !^{2} !\right)_{\leq}$-henselian.

Proof of claim. Denote by $\mathcal{O}$ the valuation ring of $v_{x}$ in $F^{\text {ra }}$. Let $g \in\left(\mathcal{O} \cap K^{\prime}\right)[X]$ be of degree $\leq n !^{2}$ !, let $a \in \mathcal{O} \cap K^{\prime}$, and suppose that $v_{x}(g(a))=0$ and $v_{x}\left(g^{\prime}(a)\right)>0$. Since $\left(F^{\mathrm{ra}}, v_{x}\right)$ is henselian, $g$ must have a root $\alpha \in F^{\mathrm{ra}}$. Clearly $\left[K^{\prime}(\alpha): K^{\prime}\right] \leq n !^{2}$ !. Since $n !^{2} !<q$, we have $\alpha \in E$. Thus $\alpha \in K^{\prime}$. This shows that $\left(K^{\prime}, v_{x}\right)$ is $\left(n !^{2} !\right)_{\leq}-$henselian.

Claim 4.8.3. $\left(K^{\prime}, v_{x}\right)$ is $n_{\leq}$-defectless.

Proof of claim. Let $(L, u) /\left(K^{\prime}, v_{x}\right)$ be an extension of degree $\leq n$. Consider the normal hull $L^{N} / K^{\prime}$ of $L / K^{\prime}$, and let $w$ denote any extension of $u$ to $L^{\bar{N}}$. It suffices to show that $\left(L^{N}, w\right) /\left(K^{\prime}, v_{x}\right)$ is defectless.

If $k$ denotes the degree $\left[L^{N}: K^{\prime}\right]$, then $k \leq n !<q$. Therefore $L^{N}$ is a subfield of $E$ and so $K^{\prime}=L^{N} \cap F^{\mathrm{ra}}$. Since $L^{N} / K^{\prime}$ is Galois, we apply Fact 4.6 to find that $L^{N} / K^{\prime}$ is linearly disjoint from $F^{\mathrm{ra}}$. Therefore $k=\left[L^{N} F^{\mathrm{ra}}: F^{\mathrm{ra}}\right]$, where $L^{N} F^{\mathrm{ra}}$ denotes the compositum of $L^{N}$ and $F^{\mathrm{ra}}$. Let $v_{x}$ also denote the unique extension of $v_{x}$ to $L^{N} F^{\mathrm{ra}}$, and its restriction from $L^{N} F^{\mathrm{ra}}$ to $L^{N}$. Since $\left(F^{\mathrm{ra}}, v_{x}\right)$ is henselian, so is $\left(L^{N} F^{\mathrm{ra}}, v_{x}\right)$.

Although the rest of this argument is standard, we include it for the convenience of the reader. The value group $v_{x} F^{\mathrm{ra}}=v_{x} K^{\prime}=\mathbb{Q}$ is divisible, and so any finite extension is trivial. Since $\left(F^{\mathrm{ra}}, v_{x}\right)$ is defectless, we have the equality:

$$
k=\left[L^{N} F^{\mathrm{ra}} v_{x}: K\right] .
$$

By the primitive element theorem and the henselianity of $\left(L^{N} F^{\mathrm{ra}}, v_{x}\right)$, there exists $b \in$ $L^{N} F^{\text {ra }}$ of degree $k$ over $K$ such that $L^{N} F^{\text {ra }} v_{x}=K\left(b v_{x}\right)$. 
Finally, we argue that $b \in L^{N}$. Since $b$ is of degree $k$ over $K^{\prime}$, we have $\left[L^{N}(b): K^{\prime}\right] \leq k^{2}$. Therefore the normal hull of $L^{N}(b) / K^{\prime}$ is an extension of degree at most $k^{2} ! \leq n !^{2} !<q$. Consequently $L^{N}(b)$ is a subfield of $E$, and so $L^{N}(b) / K^{\prime}$ is linearly disjoint from $F^{\mathrm{ra}}$. Therefore the degree $\left[L^{N}(b): K^{\prime}\right]$ is equal to the degree $k=\left[F^{\mathrm{ra}} L^{N}: F^{\mathrm{ra}}\right]$. This shows that $b$ is already an element of $L^{N}$. Thus the residue field extension $L^{N} v_{x} / K^{\prime} v_{x}$ is of degree $k$, and so $\left(L^{N}, v_{x} /\left(K^{\prime}, v_{x}\right)\right.$ is defectless. In particular, $v_{x}$ extends uniquely from $K^{\prime}$ to $L^{N}$. Thus $w=v_{x}$, and so in fact we have shown that $\left(L^{N}, w\right) /\left(K^{\prime}, v_{x}\right)$ is defectless. This completes the proof of the claim.

The theorem now follows.

4.4. Compositions of $n_{\leq}$-defectless valuations. Our next step is to prove the following lemma about compositions of $n_{\leq}$-defectless valuations. First we deal with finite compositions, and then certain infinite compositions.

Lemma 4.9. The composition of two $n_{\leq}$-defectless valuations is $n_{\leq}$-defectless. More precisely: let $u, v$ be two valuations on $K$ such that $u$ is a coarsening of $v$ let $\bar{v}$ denote the valuation induced on $K u$ by $v$, and suppose that $(K, u)$ and $(K u, \bar{v})$ are both $n_{\leq}$-defectless; then $(K, v)$ is $n_{\leq}$-defectless.

Proof. Let $\left(L, v^{\prime}\right) /(K, v)$ be an extension of degree $l \leq n$. We aim to show that this extension satisfies the fundamental equality $[L: K]=\left[L v^{\prime}: K v\right] \cdot\left(v^{\prime} L: v K\right)$.

Let $\Delta$ be the convex subgroup of $v K$ corresponding to the coarsening $u$; then $u K=$ $v K / \Delta$ and $\bar{v}(K u)=\Delta$. If $\Delta^{\prime}$ denotes the convex hull of $\Delta$ in $v^{\prime} L$, then $\Delta^{\prime}$ corresponds to the unique extension of $u$ to $L$, which we denote by $u^{\prime}$, so that $u^{\prime} L=v^{\prime} L / \Delta^{\prime}$ and $\bar{v}^{\prime}\left(L u^{\prime}\right)=\Delta^{\prime}$.

Claim 4.9.1. We have the equality $\left(v^{\prime} L: v K\right)=\left(u^{\prime} L: u K\right) \cdot\left(\Delta^{\prime}: \Delta\right)$.

In the absence of a convenient reference, we give a proof of the claim.

Proof of claim. The extensions $\left(L, v^{\prime}\right) /(K, v)$ and $\left(L, u^{\prime}\right) /(K, u)$ induce embeddings of value groups, by which we identify $u K$ with the subgroup $\left(v K+\Delta^{\prime}\right) / \Delta^{\prime}$ of $v L / \Delta^{\prime}=u^{\prime} L$. Thus the index $\left(u^{\prime} L: u K\right)$ really means the index

$$
\left(v^{\prime} L / \Delta^{\prime}:\left(v K+\Delta^{\prime}\right) / \Delta^{\prime}\right) .
$$

By the Isomorphism Theorems, there is an isomorphism

$$
v^{\prime} L /\left(v K+\Delta^{\prime}\right) \longrightarrow\left(v^{\prime} L / \Delta^{\prime}\right) /\left(\left(v K+\Delta^{\prime}\right) / \Delta^{\prime}\right) .
$$

This establishes the equality $\left(u^{\prime} L: u K\right)=\left(v^{\prime} L: v K+\Delta^{\prime}\right)$. Secondly, by another application of the Isomorphism Theorems, we have the equality $\left(v K+\Delta^{\prime}: v K\right)=\left(\Delta^{\prime}\right.$ : $\Delta$ ), since $\Delta$ is the intersection $v K \cap \Delta^{\prime}$. Putting these conclusions together and using the usual multiplicativity of indices, we have established the following

$$
\begin{aligned}
\left(v^{\prime} L: v K\right) & =\left(v^{\prime} L: v K+\Delta^{\prime}\right) \cdot\left(v K+\Delta^{\prime}: v K\right) \\
& =\left(u^{\prime} L: u K\right) \cdot\left(\Delta^{\prime}: \Delta\right) .
\end{aligned}
$$

This finishes the proof of the claim.

Since $(K, u)$ is $n_{\leq}$-defectless we have

$$
[L: K]=\left[L u^{\prime}: K u\right] \cdot\left(u^{\prime} L: u K\right) .
$$


In particular, the degree $\left[L u^{\prime}: K u\right]$ is bounded above by $[L: K]=l \leq n$. Thus $\left(L u^{\prime}, \bar{v}^{\prime}\right) /(K u, \bar{v})$ is an extension of degree $\leq n$. Since $(K u, \bar{v})$ is $n_{\leq}$-defectless we have

$$
\begin{aligned}
{\left[L u^{\prime}: K u\right] } & =\left[\left(L u^{\prime}\right) \bar{v}^{\prime}:(K u) \bar{v}\right] \cdot\left(\bar{v}^{\prime}\left(L u^{\prime}\right): \bar{v}(K u)\right) \\
& =\left[L v^{\prime}: K v\right] \cdot\left(\Delta^{\prime}: \Delta\right) .
\end{aligned}
$$

Combining these observations together with the claim, we deduce the following equality, as required:

$$
\begin{aligned}
{[L: K] } & =\left[L u^{\prime}: K u\right] \cdot\left(u^{\prime} L: u K\right) \\
& =\left[L v^{\prime}: K v\right] \cdot\left(\Delta^{\prime}: \Delta\right) \cdot\left(u^{\prime} L: u K\right) \\
& =\left[L v^{\prime}: K v\right] \cdot\left(v^{\prime} L: v K\right) .
\end{aligned}
$$

Definition 4.10. An extension $(L, w) /(K, v)$ of valued fields is immediate if both the value group extension and the residue field extension are trivial, i.e. $v K=w L$ and $K v=L w$. A valued field that admits no proper algebraic immediate extensions is said to be algebraically maximal.

Lemma 4.11. Let $K$ be a field equipped with a family of valuations $\left(v_{n}\right)_{n<\omega}$ such that the corresponding valuation rings $\left(\mathcal{O}_{n}\right)_{n<\omega}$ form an increasing chain with $K=\bigcup_{n<\omega} \mathcal{O}_{n}$. Suppose there is $k<\omega$ such that for all $n<\omega$ we have

(1) $\left(K, v_{n}\right)$ is $k_{\leq}$-henselian, and

(2) $\left(K v_{n+1}, \bar{v}_{n}\right)$ is $k_{\leq}$-defectless;

where $\bar{v}_{n}$ denotes the valuation induced on $K v_{n+1}$ by $v_{n}$.

Then, for each $n<\omega$, the valued field $\left(K, v_{0}\right)$ does not admit any non-trivial immediate extension of degree $\leq k$.

Proof. For brevity, we write $v=v_{0}$. Let $(L, w) /(K, v)$ be an immediate extension of degree $\leq k$. This means that the extension $L w / K v$ of residue fields and the extension $w L / v K$ of value groups are trivial. Our aim is to show that $[L: K]=1$, i.e. $L=K$. For each $n<\omega$, there is a unique valuation $w_{n}$ on $L$ that extends $v_{n}$ and coarsens $w$. Let $\mathcal{O}_{w_{n}}$ (respectively, $\mathfrak{m}_{w_{n}}$ ) be the valuation ring (resp., maximal ideal) of $w_{n}$. Since $L / K$ is algebraic, there is no nontrivial valuation on $L$ which is coarser than all of the valuations $w_{n}, n<\omega$. Thus we have $L=\bigcup_{n<\omega} \mathcal{O}_{w_{n}}$, and equivalently $\{0\}=\bigcap_{n<\omega} \mathfrak{m}_{w_{n}}$. Also, we denote by $\bar{w}_{n}$ the valuation induced on $L w_{n+1}$ by $w_{n}$.

We may assume that $L=K(\alpha)$, for some $\alpha \in \mathcal{O}_{w_{0}}$. Let $f \in K[X]$ be the minimal polynomial of $\alpha$ over $K$. Since $K$ is perfect, $f$ is separable. Thus $f(\alpha)=0$ and $D f(\alpha) \neq 0$, where $D f$ denotes the formal derivative of $f$.

We choose $N<\omega$ large enough such that $f \in \mathcal{O}_{v_{N}}[X]$. and $D f(\alpha) \notin \mathfrak{m}_{w_{N}}$. Applying the residue maps of $v_{N}$ and $w_{N}$, we have

$$
\left(D f v_{N}\right)\left(\alpha w_{N}\right)=D f(\alpha) w_{N} \neq 0
$$

and trivially $\left(f v_{N}\right)\left(\alpha w_{N}\right)=f(\alpha) w_{N}=0$. Thus $\alpha w_{N} \in L w_{N}$ is a simple root of $f v_{N}$.

Consider the compositions $\tilde{v}:=\bar{v}_{N-1} \circ \ldots \circ \bar{v}_{0}$ and $\tilde{w}:=\bar{w}_{N-1} \circ \ldots \circ \bar{w}_{0}$. By Lemma 4.9, the composition of finitely many $k_{\leq}$-defectless valuations is $k_{\leq}$-defectless. Thus $\left(K v_{N}, \tilde{v}\right)$ is $k_{\leq}$-defectless, and so the fundamental equality

$$
\left[L w_{N}: K v_{N}\right]=\left[\left(L w_{N}\right) \tilde{w}:\left(K v_{N}\right) \tilde{v}\right] \cdot\left(\tilde{w}\left(L w_{N}\right): \tilde{v}\left(K v_{N}\right)\right)
$$

holds for the extension $\left(L w_{N}, \tilde{w}\right) /\left(K v_{N}, \tilde{v}\right)$. The value groups $\tilde{w}\left(L w_{N}\right)$ and $\tilde{v}\left(K v_{N}\right)$ are in fact the same convex subgroup of $w L=v K$, and thus the extension of value groups is trivial. Therefore, we have

$$
\left[L w_{N}: K v_{N}\right]=\left[\left(L w_{N}\right) \tilde{w}:\left(K v_{N}\right) \tilde{v}\right]=[L w: K v]=1
$$


by our assumption that $(L, w) /(K, v)$ is immediate. Therefore $L w_{N}=K v_{N}$.

Putting all of this together, $\alpha w_{N} \in K v_{N}$ is a simple root of $f v_{N}$. Since $\left(K, v_{N}\right)$ is $k_{\leq^{-}}$ henselian by assumption, there exists $a \in \mathcal{O}_{N} \subseteq K$ such that $a v_{N}=\alpha w_{N}$ and $f(a)=0$. This shows that $[L: K]=1$, as required.

\subsection{The construction, stage II.}

Lemma 4.12. For each $n<\omega$, there exists a sentence $\pi_{n}$ in the language of valued fields

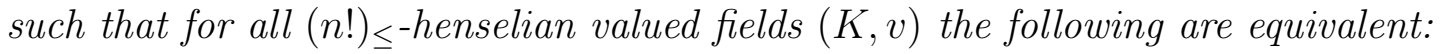

(1) $(K, v) \models \pi_{n}$

(2) $(K, v)$ admits no proper immediate extensions of degree $\leq n$.

Proof. We follow closely the idea of [Kuh10, Proposition 6.3], and we assume some familiarity with Kaplansky's theory of pseudo-Cauchy sequences (also called 'pseudoconvergent' sequences) from Kap42. It is clear that the property 'for all $f \in K[X]$ of degree $\leq n$ the set $v \operatorname{im}_{K}(f)=\{v(f(a)) \mid a \in K\}$ has a maximum' is expressible by a sentence in the language of valued fields. Choose $\pi_{n}$ to be any such sentence.

Let $(K, v)$ be an $(n !)_{\leq}$-henselian valued field. From [FJ15, Lemma 6.3] it follows that $v$ extends uniquely to every Galois extension of degree $\leq n$ !. Therefore $v$ extends uniquely to every extension of degree $\leq n$.

$(1 \Longrightarrow 2)$ Suppose that $(L, w) /(K, v)$ is a proper immediate extension of degree $\leq n$. Let $a \in L \backslash K$. By [Kap42, Theorem 1], there is a pseudo-Cauchy sequence $\left(c_{\nu}\right)_{\nu<\lambda}$ in $K$ without a limit in $K$ of which $a$ is a limit. Let $f \in K[X]$ be the minimal polynomial of $a$ over $K$. Of course $\operatorname{deg}(f) \leq n$. As argued above, the extension of $v$ to $L$ is unique. Thus we may apply [Kuh10, Lemma 2.11] to find that $v \operatorname{im}_{K}(f)$ has no maximum.

$(1 \Longleftarrow 2)$ Let $f \in K[X]$ be of degree $\leq n$, and suppose that $v \operatorname{im}_{K}(f)$ has no maximum. In particular $f$ is of degree $\geq 2$. By [Kuh10, Lemma 6.1], there is a pseudo-Cauchy sequence $\left(c_{\nu}\right)_{\nu<\lambda}$ in $K$ which is of algebraic type, is without a limit in $K$, and is such that $\left(f\left(c_{\nu}\right)\right)_{\nu<\lambda}$ is a strictly increasing cofinal sequence in $v \operatorname{im}_{K}(f)$. In particular, the polynomial $f$ witnesses that $\left(c_{\nu}\right)_{\nu<\lambda}$ is of algebraic type. Let $g$ be the polynomial of minimum degree that witnesses that $\left(c_{\nu}\right)_{\nu<\lambda}$ is of algebraic type. Then $g$ is irreducible and we have $2 \leq \operatorname{deg}(g) \leq \operatorname{deg}(f)$. By [Kap42, Theorem 3], $(K, v)$ admits a proper immediate extension generated by a root of $g$.

This proves the stated equivalence.

Finally we are ready for the final stage of the construction.

Proposition 4.13. Let $p$ be a prime or zero. There exists a non-henselian t-henselian field of characteristic $p$ of divisible-tame type which is not separably closed.

This proposition is our version of [FJ15, Construction 6.5], which uses our Lemma 4.8 instead of [FJ15, Lemma 6.4]. As such, our proof is very similar to that of [FJ15, Construction 6.5]. Nevertheless, we go into some detail in order to be able to highlight the points of difference.

Proof. Let $K_{0}$ be any field of characteristic $p$ which is perfect and contains all roots of unity. For each $n<\omega$, write $k_{n}:=n+p+1$ and choose a prime $q_{n}$ such that $k_{n} !^{2} !<q_{n}$.

We apply Lemma 4.8 to obtain a valued field $\left(K_{1}, \bar{v}_{0}\right)$ which is not $q_{0}$-henselian, but is $\left(k_{0} !^{2} !\right)_{\leq}$-henselian, and is $\left(k_{0}\right)_{\leq}$-defectless. Also $K_{1}$ is of characteristic $p$ and is perfect and contains all roots of unity. Finally, the residue field $K_{1} \bar{v}_{0}$ is $K_{0}$, and the value group $\bar{v}_{0} K_{1}$ is $\mathbb{Q}$. 
We continue to apply Lemma 4.8 recursively. In this way we obtain a sequence $\left(K_{n+1}, \bar{v}_{n}\right)_{n<\omega}$ of valued fields with the corresponding places forming a chain:

$$
\ldots \rightarrow K_{n} \stackrel{\bar{v}_{n-1}}{\rightarrow-\rightarrow} K_{n-1} \rightarrow \ldots K_{1} \stackrel{\bar{v}_{0}}{\rightarrow} K_{0}
$$

such that each $\left(K_{n+1}, \bar{v}_{n}\right)$ is not $q_{n}$-henselian, but is $\left(k_{n} !^{2} !\right)_{\leq}$-henselian, and is $\left(k_{n}\right)_{\leq^{-}}$ defectless. Moreover $\left(K_{n+1}, \bar{v}_{n}\right)$ has residue field $K_{n+1} \bar{v}_{n}=K_{n}$ and value group $\bar{v}_{n} K_{n+1}=$ $\mathbb{Q}$.

For $n>m$, there is the composition $v_{n, m}:=\bar{v}_{m} \circ \ldots \circ \bar{v}_{n-1}$. This is a nontrivial valuation on $K_{n}$ with residue field $K_{n} v_{n, m}=K_{m}$ and value group $v_{n, m} K_{n} \equiv \mathbb{Q}$. We denote by $\mathcal{O}_{n, m}$ the valuation ring corresponding to $v_{n, m}$, and we write $\mathcal{O}_{0,0}=K_{0}$. Then for $n>m$ the residue map $\mathcal{O}_{n, m} \longrightarrow K_{m}$ restricts to a ring epimorphism $\pi_{n, m}: \mathcal{O}_{n, 0} \longrightarrow \mathcal{O}_{m, 0}$. Thus the rings $\left(\mathcal{O}_{n, 0}\right)_{n<\omega}$ together with the maps $\left(\pi_{n, m}\right)_{m<n}$ form a projective system.

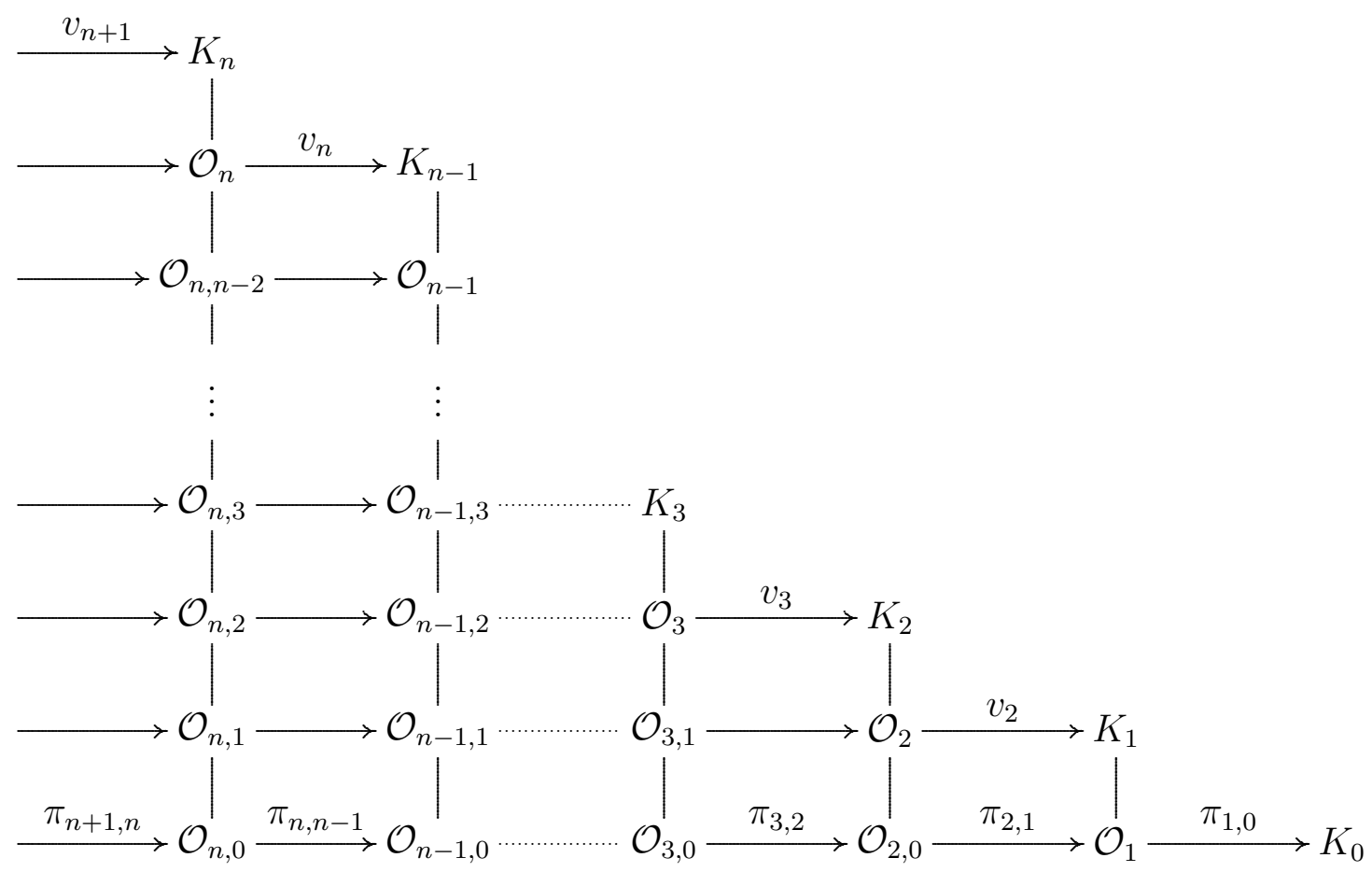

Figure 2: The projective system

Let $\mathcal{O}$ together with the natural projections $\pi_{\infty, n}: \mathcal{O} \longrightarrow \mathcal{O}_{n, 0}$ be the projective limit of this system, and let $K$ be the quotient field of $\mathcal{O}$. By [FP11, Lemma 3.5], $\mathcal{O}$ is a valuation ring. For each $n<\omega$, let $\mathfrak{p}_{n}$ denote the kernel of $\pi_{\infty, n}$, and let $\mathcal{O}_{\mathfrak{p}_{n}}$ denote the localisation of $\mathcal{O}$ at $\mathfrak{p}_{n}$. Since $\mathfrak{p}_{n} \supseteq \mathfrak{p}_{n+1}$, we have $\mathcal{O}_{\mathfrak{p}_{n}} \subseteq \mathcal{O}_{\mathfrak{p}_{n+1}}$. Since $\{0\}=\bigcap_{n<\omega} \mathfrak{p}_{n}$, we have $K=\bigcup_{n<\omega} \mathcal{O}_{\mathfrak{p}_{n}}$.

Let $v_{n}$ denote the valuation on $K$ with valuation $\operatorname{ring} \mathcal{O}_{\mathfrak{p}_{n}}$. Then $\left(v_{n}\right)_{n<\omega}$ is a strictly increasing (i.e. increasingly coarse) chain of valuations on $K$; and the finest common coarsening of this chain is the trivial valuation. Moreover, each $v_{n}$ induces the valuation $\bar{v}_{n}$ on the residue field $K v_{n+1}=K_{n+1}$. For each $n<\omega$, the value group $v_{n} K$ is the directed union of the convex subgroups $v_{m, n} K_{m}$, for $m>n$; and each of these subgroups is nontrivial and divisible. Thus $v_{n} K$ is also nontrivial and divisible.

By the argument in the first paragraph of the proof of [FJ15, Proposition 6.7], $\left(K, v_{n}\right)$ is $\left(k_{n} !^{2}\right)_{\leq}$-henselian. Trivially this implies that $\left(K, v_{n}\right)$ is $\left(k_{n} !\right)_{\leq-}$-henselian and $\left(k_{n}\right)_{\leq-}$ henselian. 
Now, for each $n<\omega$, we have shown that the hypotheses of Lemma 4.11 are satisfied by $K$ together with the family $\left(v_{m+n}\right)_{m<\omega}$, for $k=k_{n}$. Therefore $\left(K, v_{n}\right)$ does not admit any non-trivial immediate extensions of degree $\leq k_{n}$. By Lemma 4.12, we have $\left(K, v_{n}\right) \models \pi_{k_{n}}$.

Let $\left(K^{*}, v^{*}\right)$ be the ultraproduct of the family $\left(K, v_{n}\right)_{n<\omega}$ with respect to a non-principal ultrafilter. By Łoś's Theorem [Hod97, Theorem 8.5.3], $\left(K^{*}, v^{*}\right)$ is a perfect equicharacteristic nontrivially valued field with divisible value group. Since ' $n_{\leq}$-henselianity' is an elementary property of valued fields, again by Łos's Theorem, we have that $\left(K^{*}, v^{*}\right)$ is $n_{\leq}$-henselian, for all $n<\omega$. Thus $\left(K^{*}, v^{*}\right)$ is henselian. By yet another application of Eoś's Theorem, $\left(K^{*}, v^{*}\right)=\pi_{n}$, for each $n<\omega$. Since $\left(K^{*}, v^{*}\right)$ is also henselian, we have that $\left(K^{*}, v^{*}\right)$ is algebraically maximal, by Lemma 4.12. In [Kuh16, Corollary 3.4a] it is shown that a perfect equicharacteristic valued field which is algebraically maximal is in fact henselian and defectless. In particular $\left(K^{*}, v^{*}\right)$ is tame. Therefore $K$ is a t-henselian field of divisible-tame type.

The field $K$ is non-henselian by exactly the same arguments as in [FJ15, Proposition 6.7]: if $w$ were an henselian valuation on $K$ then $w$ would be a coarsening of $v_{n}$, for each $n<\omega$, but their least common coarsening is trivial. Note that since $K$ admits nontrivial non-henselian valuations, $K$ is not separably closed. Therefore $K$ is a non-henselian thenselian field of characteristic $p$ of divisible-tame type which is not separably closed, as required.

\section{FIELDS OF MIXED-CHARACTERISTIC}

The goal of this section is to prove part (B) of Theorem 1.1. We've already seen in Corollary 2.2 that (mc) implies (eh). This leaves us with showing for mixed characteristic fields that

(1) (h) does not imply ( $\emptyset$-def), and

(2) (def) implies ( $\emptyset$-def).

5.1. Self-similarity. As a preliminary to deducing (1) in Example 5.5, we first adapt the Ax-Kochen/Ershov argument from the proof of Lemma 3.2 to the slightly more general setting of $t$-henselian fields of divisible-tame type.

Lemma 5.1. Let $k$ be a t-henselian field of divisible-tame type. Then $k \preceq k\left(\left(t^{\mathbb{Q}}\right)\right)$.

Proof. By definition of 'divisible-tame type' there exists a field $K \equiv k$ and a nontrivial valuation $v$ on $K$ such that $(K, v)$ is tame and $v K$ is divisible. Using the compactness theorem, we may assume that $v$ is equicharacteristic and that $K$ is an elementary extension of $k$. Moreover, both $K$ and $k$ are perfect.

Consider the field $K\left(\left(t^{\mathbb{Q}}\right)\right)$ together with the valuation $u$, which we define to be the composition $v \circ v_{t}$ of $v$ with the $t$-adic valuation $v_{t}$. Then $\left(K\left(\left(t^{\mathbb{Q}}\right)\right), u\right) /(K, v)$ is an extension of equicharacteristic tame valued fields. The extension of residue fields is the identity map $K v \longrightarrow K v$, which is elementary. Moreover the extension of value groups is elementary since the theory of nontrivial divisible ordered abelian groups is modelcomplete. By the $\mathrm{AKE}^{\prec}$-principle for the theory of tame valued fields (see [Kuh16, Theorem 1.4]), we have that $(K, v) \preceq\left(K\left(\left(t^{\mathbb{Q}}\right)\right), u\right)$ is an elementary extension of valued fields.

Since $k$ is perfect, $\left(k\left(\left(t^{\mathbb{Q}}\right)\right), v_{t}\right)$ is tame. By another application of the AKE ${ }^{\preceq}$-principle for tame valued fields, $\left(k\left(\left(t^{\mathbb{Q}}\right)\right), v_{t}\right) \preceq\left(K\left(\left(t^{\mathbb{Q}}\right)\right), v_{t}\right)$ is also an elementary extension of valued fields. In particular, both $K \preceq K\left(\left(t^{\mathbb{Q}}\right)\right)$ and $k\left(\left(t^{\mathbb{Q}}\right)\right) \preceq K\left(\left(t^{\mathbb{Q}}\right)\right)$ are elementary 
extensions of fields. Finally we consider the following diagram of field extensions.

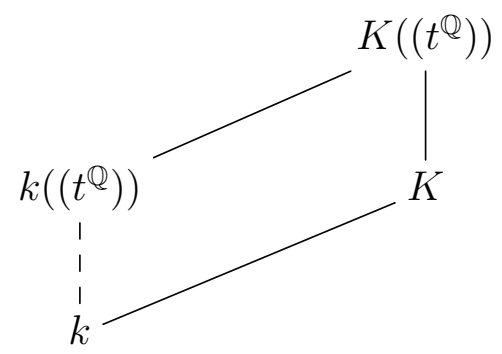

Since each solid line represents an elementary extension of fields, the dotted line is also elementary. Thus $k \preceq k\left(\left(t^{\mathbb{Q}}\right)\right)$ is elementary, as required.

Of course, in mixed-characteristic, a field cannot be elementarily equivalent to its residue field, simply for reasons of characteristic. Instead, we give the following definition.

Definition 5.2. We say a valued field $(L, w)$ is self-similar if there is an elementary extension $\left(L^{*}, w^{*}\right) \succeq(L, w)$ and a valuation $u$ on $L^{*}$ which is not equivalent to $w^{*}$ such that $\left(L^{*}, w^{*}\right) \equiv\left(L^{*}, u\right)$.

It is clear that if $(L, w)$ is self-similar then $w$ cannot be $\emptyset$-definable.

Proposition 5.3. Let $p$ be any prime, let $k$ be a t-henselian field of characteristic $p$ of divisible-tame type, and let $(L, w)$ be a mixed-characteristic tame valued field with $w L \equiv \mathbb{Q}$ and $L w=k$. Then $(L, w)$ is self-similar.

Proof. Let $\left(L^{\prime}, w^{\prime}\right)$ be any extension of $(L, w)$ such that $w^{\prime} L^{\prime}$ is divisible, and the residue field extension $L^{\prime} w^{\prime} / L w$ is $k\left(\left(t^{\mathbb{Q}}\right)\right) / k$. For example, to find such $\left(L^{\prime}, w^{\prime}\right)$ we can apply [Kuh04, Theorem 2.14].

Let $(\hat{L}, \hat{w})$ be a maximal immediate extension of $\left(L^{\prime}, w^{\prime}\right)$. Then $\hat{L}$ is henselian and defectless, with nontrivial divisible value group, and perfect residue field $k\left(\left(t^{\mathbb{Q}}\right)\right)$. Thus $(\hat{L}, \hat{w})$ is a mixed-characteristic tame valued field. Consider the extension $(\hat{L}, \hat{w}) /(L, w)$. By the model completeness of the theory of nontrivial divisible ordered abelian groups, the extension of value groups is elementary. By an application of Lemma 5.1, the extension of residue fields is also elementary. Therefore, by the $\mathrm{AKE}^{-}$-principle for tame valued fields (see [Kuh16, Theorem 1.4]), $(L, w) \preceq(\hat{L}, \hat{w})$ is an elementary extension.

Next we let $u$ be the composition $v_{t} \circ \hat{w}$. Then $(\hat{L}, u)$ is henselian and defectless. The value group $u \hat{L}$ is an extension of $\mathbb{Q}$ by a nontrivial divisible ordered abelian group, thus $u \hat{L}$ is divisible. By another application of the model completeness of the theory of nontrivial divisible ordered abelian groups, the extension of value groups is elementary. The residue field $\hat{L} u$ is $k$, and the extensions of residue fields is simply the identity map $k \longrightarrow k$. In particular, the extension of residue fields is elementary. Therefore, by another application of the $\mathrm{AKE}^{\prec}$-principle for tame valued fields (see [Kuh16, Theorem $1.4]),(L, w) \preceq(\hat{L}, u)$ is an elementary extension.

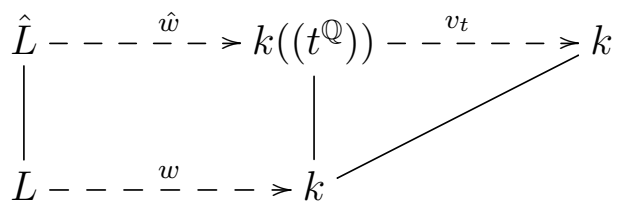

We have shown that both $(\hat{L}, \hat{w})$ and $(\hat{L}, u)$ are elementary extensions of $(L, w)$. Since $\hat{w}$ is a proper coarsening of $u$, we conclude that $(L, w)$ is self-similar. 
5.2. 'Henselian' does not imply 'definable'. One of the remaining questions in mixed characteristic (short of giving a characterisation of fields with (def)) is whether or not all fields in $\mathcal{K}_{0, p}$ admit definable nontrivial henselian valuations. The answer is 'not'. For any prime $p$, we exhibit in Example 5.5 a field in $\mathcal{K}_{0, p}$ which does not admit a $\emptyset$-definable nontrivial henselian valuation. By|Theorem 5.7, these fields do not even admit a definable nontrivial henselian valuation.

Proposition 5.4. Let $(L, w)$ be a tame valued field of mixed characteristic such that $w L=\mathbb{Q}$ and $L w$ is a non-henselian t-henselian field of divisible-tame type which is not separably closed. Then $L$ does not admit a $\emptyset$-definable nontrivial henselian valuation.

Proof. The residue field $L w$ is not separably closed by assumption, therefore all henselian valuations on $L$ are comparable to $w$. Moreover, we have assumed that $L w$ is nonhenselian, thus no proper refinement of $w$ is henselian. Also, the value group $w L$ is archimedean, so there are no nontrivial proper coarsenings of $w$. Putting these three facts together we deduce that $w$ is the only nontrivial henselian valuation on $L$.

Next we note that $(L, w)$ satisfies the hypotheses of Proposition 5.3, from which we conclude that $(L, w)$ is self-similar, This means that there exists an elementary extension $(L, w) \preceq\left(L^{*}, w^{*}\right)$ and a valuation $u$ on $L^{*}$, which is different from $w^{*}$, such that $\left(L^{*}, w^{*}\right) \equiv$ $\left(L^{*}, u\right)$. Consequently, $w^{*}$ is not $\emptyset$-definable in $L^{*}$, and $w$ is not $\emptyset$-definable in $L$. Since $L$ admits no other nontrivial henselian valuation, as argued above, we conclude that $L$ admits no $\emptyset$-definable nontrivial henselian valuation.

Example 5.5. We are now able to exhibit the promised example to demonstrate that 'henselian' does not imply 'definable' for fields in $\mathcal{K}_{0, p}$. Let $p$ be any prime and let $k$ be a non-henselian t-henselian field of characteristic $p$ of divisible-tame type which is not separably closed. For example we may choose $k$ to be any field constructed by Proposition 4.13. By [Kuh16, Lemma 3.1], tame valued fields are perfect. Since $k$ is elementarily equivalent to a field that admits a nontrivial tame valuation, $k$ is also perfect. Let $(L, w)$ be a mixed-characteristic tame valued field with $w L=\mathbb{Q}$ and $L w=k$. For example, we start with the valued field of $p$-adic numbers $\left(\mathbb{Q}_{p}, v_{p}\right)$. Then, by $[\mathrm{Kuh04}$, Theorem 2.14], there exists an extension $(K, v) /\left(\mathbb{Q}_{p}, v_{p}\right)$ such that $v K=\mathbb{Q}$ and $K v \cong k$. In particular $(K, v)$ is of mixed characteristic. Now let $(L, w)$ be a maximal immediate extension of $(K, v)$. Since $(L, w) /(K, v)$ is immediate, $w L=\mathbb{Q}$ and $L w \cong k$. By Kuh16, Theorem 3.2], $(L, w)$ is tame. Applying Proposition 5.4 to $L$, we conclude that $L$ does not admit any $\emptyset$-definable nontrivial henselian valuation.

Since $L w \cong k$ is not separably closed, $v_{L}$ is a refinement of $w$. Consequently, the characteristic of $L v_{L}$ is also $p$. Since also the characteristic of $L$ is zero, we conclude that $L \in \mathcal{K}_{0, p}$, as required.

5.3. 'Definable' implies ' $\emptyset$-definable'. The aim of this subsection is to show that for any prime $p$ and any $K \in \mathcal{K}_{0, p}$, we have

$$
(\text { def }) \Longrightarrow(\emptyset \text {-def })
$$

The proof uses the machinery of $q$-henselian valuations as developed in [JK15b]. Let $q$ be any prime. Recall that a valuation $v$ on a field $L$ is called $q$-henselian if $v$ extends uniquely to every Galois extension of $L$ of $q$-power degree. Let $L$ be a field admitting nontrivial Galois extensions of $q$-power degree; we denote this by $L \neq L(q)$. Then, there is always a canonical $q$-henselian valuation $v_{L}^{q}$, and the definiton is similar to that of the canonical henselian valuation. Again, we divide the class of $q$-henselian valuations on $L$ into two subclasses, namely

$$
H_{1}^{q}(L)=\{v q \text {-henselian on } L \mid L v \text { admits a Galois extension of degree } q\}
$$


and

$H_{2}^{q}(L)=\{v q$-henselian on $L \mid L v$ does not admit a Galois extension of degree $q\}$.

One can deduce that any valuation $v_{2} \in H_{2}^{q}(L)$ is strictly finer than any $v_{1} \in H_{1}^{q}(L)$, i.e. $\mathcal{O}_{v_{2}} \subsetneq \mathcal{O}_{v_{1}}$, and that any two valuations in $H_{1}^{q}(L)$ are comparable. Furthermore, if $H_{2}^{q}(L)$ is non-empty, then there exists a unique coarsest valuation $v_{L}^{q}$ in $H_{2}^{q}(L)$; otherwise there exists a unique finest valuation $v_{L}^{q} \in H_{1}^{q}(L)$. In either case, $v_{L}^{q}$ is called the canonical $q$-henselian valuation. Note that any henselian valuation on $L$ is $q$-henselian and thus comparable to $v_{L}^{q}$ We denote by $\mathcal{O}_{L}^{q}$ the valuation ring of $v_{L}^{q}$, and continue to denote by $\mathcal{O}_{L}$ the valuation ring of the canonical henselian valuation $v_{L}$ on $L$.

Our proof uses a special case of the uniform definability of canonical $q$-henselian valuation as proven in [JK15b, Main Theorem]: Let $\mathcal{F}_{q}$ be the (elementary) class of fields $L$ such that $L \neq L(q)$, and that $L$ contains a primitive $q$ th root of unity $\zeta_{q}$ in case $\operatorname{char}(L) \neq q$. In case $q=2$, assume further that $L$ is non-orderable. There is a parameter-free $\mathcal{L}_{\text {ring- }}{ }^{-}$ formula $\varphi_{q}(x)$ such that we have

$$
L \in \mathcal{F}_{q} \Longrightarrow \varphi_{q}(L)=\mathcal{O}_{L}^{q} .
$$

Furthermore, we will make repeated use of the following

Fact 5.6 ([EP05, p. 43 and Corollary 4.1.4]). Let $\mathcal{O} \subseteq K$ be a valuation ring. The overrings of $\mathcal{O}$ in $K$ form a chain under inclusion and each overring is a valuation ring. If $\mathcal{O}$ is henselian, then all overrings of $\mathcal{O}$ in $K$ are henselian.

We can now prove the main result of this subsection:

Theorem 5.7. If $\left(K, v_{K}\right)$ has mixed-characteristic then

$$
(\text { def }) \Longrightarrow(\emptyset \text {-def }) \text {. }
$$

Proof. Fix a prime $p$. Let $K$ be a field with $\operatorname{char}(K)=0$ and $\operatorname{char}\left(K v_{K}\right)=p>0$ which admits a definable nontrivial henselian valuation. Then $v_{K}$ is also nontrivial, since $\left(K, v_{K}\right)$ has mixed characteristic. Since $K$ admits a definable nontrivial henselian valuation, it is not separably closed. Furthermore, by [JK15a, Theorem A], we may assume that $K v_{K} \neq K v_{K}^{\text {sep }}$. Since $K$ is not separably closed, there exists a prime $q$ and a finite extension $L_{0} / K$ such that $L_{0} \neq L_{0}(q)$ and $\zeta_{q} \in L_{0}$. Let $n:=\left[L_{0}: K\right]$ and define

$$
\mathcal{L}:=\left\{L \mid[L: K]=n, L \neq L(q), \zeta_{q} \in L\right\} .
$$

Observe that for any field $L, L \neq L(q)$ implies that $L$ admits a Galois extension of degree $q$, by Sylow's Theorems. Therefore the family $\mathcal{L}$ is uniformly interpretable in $K$ : we quantify over those $n$-tuples from $K$ which are the coefficients of irreducible polynomials over $K$, such polynomials are the minimal polynomials of generators of extensions $L / K$, and we can define those tuples corresponding to extensions $L \in \mathcal{L}$, using the observation.

Next we explain a few basic facts about the canonical $q$-henselian valuations $v_{L}^{q}$ that we will repeatedly use. Let $L \in \mathcal{L}$. Since $L / K$ is a finite extension and $K v_{K}$ is not separably closed, $v_{L}$ is the unique extension of $v_{K}$ to $L$. Since $v_{L}^{q}$ is comparable to $v_{L},\left.v_{L}^{q}\right|_{K}$ is also comparable to $v_{K}$. Again, since $v_{L}^{q}$ is comparable to $v_{L}$, the residue characteristic of $v_{L}^{q}$ is either 0 or $p$. Finally, since $v_{L}$ is a nontrivial $q$-henselian valuation and $L \neq L(q)$, we have that $v_{L}^{q}$ and thus $\left.v_{L}^{q}\right|_{K}$ are nontrivial.

We define

$$
\mathcal{L}_{1}:=\left\{L \in \mathcal{L} \mid \operatorname{char}\left(L v_{L}^{q}\right)=p\right\}
$$

and

$$
\mathcal{L}_{2}:=\left\{L \in \mathcal{L} \mid \operatorname{char}\left(L v_{L}^{q}\right) \neq p\right\}=\left\{L \in \mathcal{L} \mid \operatorname{char}\left(L v_{L}^{q}\right)=0\right\}
$$


Just as for $\mathcal{L}$ above, both $\mathcal{L}_{1}$ and $\mathcal{L}_{2}$ are uniformly interpretable in $K$. To see that $\mathcal{L}_{1}$ is uniformly interpretable: given a uniform interpretation of $\mathcal{L}$, we then need to define which $n$-tuples correspond to extensions $L / K$ such that $\operatorname{char}\left(L v_{L}^{q}\right)=p$, and this follows from the fact that $v_{L}^{q}$ is uniformly $\emptyset$-definable in $L$, using the formula $\varphi_{q}(x)$, by [JK15b, Main Theorem]. Let $\Lambda_{1}(\mathbf{y})$ and $\Lambda_{2}(\mathbf{y})$ be the formulas that define those $n$-tuples corresponding to extensions $L / K$ in $\mathcal{L}_{1}$ and $\mathcal{L}_{2}$, respectively.

Furthermore, using the uniform interpretation of $\mathcal{L}$, we may find a formula $\phi_{q}(x, \mathbf{y})$ such that if $\mathbf{b} \in \Lambda_{1}(K) \cup \Lambda_{2}(K)$ defines a field $L \in \mathcal{L}_{1} \cup \mathcal{L}_{2}$ then $\phi_{q}(x, \mathbf{b})$ defines in $K$ the intersection $\varphi_{q}(L) \cap K=\mathcal{O}_{L}^{q} \cap K$.

We proceed by a case distinction. In each case our goal is of course to find an $\emptyset$-definable nontrivial henselian valuation on $K$.

Case 1: Suppose first that $\mathcal{L}_{2} \neq \emptyset$ and let $L \in \mathcal{L}_{2}$. As noted above, $\mathcal{O}_{L}^{q}$ is comparable to $\mathcal{O}_{L}$. Since $L \in \mathcal{L}_{2}$, $\operatorname{char}\left(L v_{L}^{q}\right)=0$. Thus $\mathcal{O}_{L} \subset \mathcal{O}_{L}^{q}$ and $\mathcal{O}_{K}=\mathcal{O}_{L} \cap K \subset \mathcal{O}_{L}^{q} \cap K$. We have the following diagram.

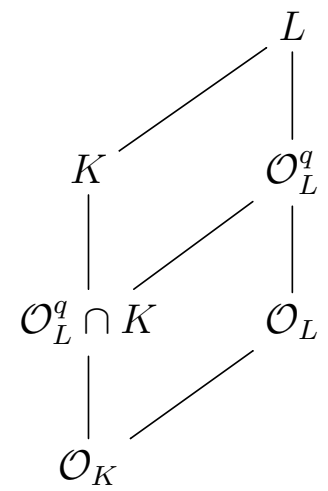

We let

$$
\mathcal{O}_{1}:=\bigcap_{L \in \mathcal{L}_{2}} \mathcal{O}_{L}^{q} \cap K
$$

It is immediate that $\mathcal{O}_{K} \subseteq \mathcal{O}_{1}$. By Fact 5.6, $\mathcal{O}_{1}$ is an henselian valuation ring. As noted above, each $\mathcal{O}_{L}^{q} \cap K$ is nontrivial. Since $\mathcal{O}_{1} \subseteq \mathcal{O}_{L}^{q} \cap K$, for each $L \in \mathcal{L}_{2}, \mathcal{O}_{1}$ is also nontrivial.

Finally, $\mathcal{O}_{1}$ is $\emptyset$-defined in $K$ by the formula

$$
\forall \mathbf{y}\left(\Lambda_{2}(\mathbf{y}) \longrightarrow \phi_{q}(x, \mathbf{y})\right) \text {. }
$$

Case 2: Now suppose that $\mathcal{L}_{2}=\emptyset$. Let $\phi(x, \mathbf{t})$ be an $\mathcal{L}_{\text {ring }}$-formula with parameters $\mathbf{t} \in K^{n}$, for some $n \in \omega$, that defines in $K$ a nontrivial henselian valuation ring $\mathcal{O}_{\mathbf{t}}$, i.e. $\phi(K, \mathbf{t})=\mathcal{O}_{\mathbf{t}}$.

For $L \in \mathcal{L}$, let $\mathcal{O}_{\mathbf{t}, L}$ denote the unique extension of $\mathcal{O}_{\mathbf{t}}$ to $L$. Then $\mathcal{O}_{\mathbf{t}, L}$ is henselian, thus $q$-henselian. Therefore $\mathcal{O}_{\mathbf{t}, L}$ is comparable to $\mathcal{O}_{L}^{q}$, and so their restrictions to $K$ (which are $\mathcal{O}_{\mathbf{t}}$ and $\mathcal{O}_{L}^{q} \cap K$ ) are comparable. Therefore

$$
\mathcal{L}_{1}=\left\{L \in \mathcal{L}_{1} \mid \mathcal{O}_{L}^{q} \cap K \subseteq \mathcal{O}_{\mathbf{t}}\right\} \sqcup\left\{L \in \mathcal{L}_{1} \mid \mathcal{O}_{\mathbf{t}} \subset \mathcal{O}_{L}^{q} \cap K\right\}
$$

This allows us to distinguish two subcases: in Case 2a, for some $L \in \mathcal{L}_{1}$ the $\operatorname{ring} \mathcal{O}_{L}^{q} \cap K$ is a strict coarsening of $\mathcal{O}_{\mathbf{t}}$; whereas in Case $\mathbf{2 b}$, for every $L \in \mathcal{L}_{1}$ the ring $\mathcal{O}_{L}^{q} \cap K$ is a refinement of $\mathcal{O}_{\mathbf{t}}$.

In the meantime, we let $S:=\bigcup_{L \in \mathcal{L}_{1}} \mathcal{O}_{L}^{q} \cap K$, and note that $S$ is $\emptyset$-defined in $K$ by the formula

$$
\exists \mathbf{y}\left(\Lambda_{1}(\mathbf{y}) \wedge \phi_{q}(x, \mathbf{y})\right) \text {. }
$$

As $S$ is a union of valuation rings each of which is comparable to $\mathcal{O}_{\mathbf{t}}, S$ is also comparable to $\mathcal{O}_{\mathbf{t}}$. In fact, in Case 2a, we have $\mathcal{O}_{\mathbf{t}} \subset S$; and in Case $\mathbf{2 b}$, we have $S \subseteq \mathcal{O}_{\mathbf{t}}$. 
From now on we separate the subcases.

Case 2a: We suppose that for some $L^{\prime} \in \mathcal{L}_{1}$ the $\operatorname{ring} \mathcal{O}_{L^{\prime}}^{q} \cap K$ is a strict coarsening of $\mathcal{O}_{\mathbf{t}}$. If we let $\mathcal{L}_{1}^{\prime}:=\left\{L \in \mathcal{L}_{1} \mid \mathcal{O}_{\mathbf{t}} \subset \mathcal{O}_{L}^{q} \cap K\right\}$ then our assumption may be rephrased as $\mathcal{L}_{1}^{\prime} \neq \emptyset$. We will show that $S$ is a mixed characteristic nontrivial henselian valuation ring, and we already know that $S$ is $\emptyset$-definable in $K$. Note that, as discussed above, in this subcase we have $\mathcal{O}_{\mathbf{t}} \subset S$, although we do not make direct use of this fact.

For each $L \in \mathcal{L}_{1} \backslash \mathcal{L}_{1}^{\prime}$, we have

$$
\mathcal{O}_{L}^{q} \cap K \subseteq \mathcal{O}_{\mathbf{t}} \subset \mathcal{O}_{L^{\prime}}^{q} \cap K \subseteq S
$$

Consequently

$$
S=\bigcup_{L \in \mathcal{L}_{1}} \mathcal{O}_{L}^{q} \cap K=\bigcup_{L \in \mathcal{L}_{1}^{\prime}} \mathcal{O}_{L}^{q} \cap K
$$

and therefore $S$ is a union of valuation rings each of which is a strict coarsening of $\mathcal{O}_{\mathbf{t}}$.

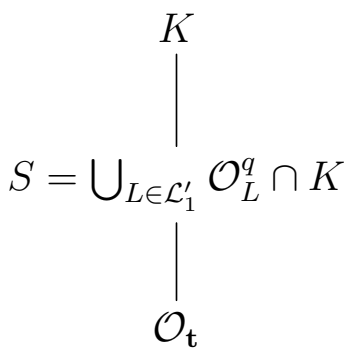

By Fact 5.6, the coarsenings of $\mathcal{O}_{\mathbf{t}}$ form a chain under inclusion, and so $S$ is a union of a chain of valuation rings. Therefore $S$ is a valuation ring. Since $S$ coarsens $\mathcal{O}_{\mathbf{t}}, S$ is henselian. Finally, since $S$ is a union of mixed characteristic valuation rings, $S$ has mixed characteristic. In particular, $S$ is nontrivial.

Case 2b: We suppose that for every $L \in \mathcal{L}_{1}$ the $\operatorname{ring} \mathcal{O}_{L}^{q} \cap K$ is a refinement of $\mathcal{O}_{\mathbf{t}}$. As noted above, we have $S \subseteq \mathcal{O}_{\mathbf{t}}$.

Since $S$ contains a valuation ring (e.g. $\mathcal{O}_{L}^{q} \cap K$, for any $L \in \mathcal{L}_{1}$ ), the set of subrings of $K$ which contain $S$ is totally ordered, by Fact 5.6. Therefore, any (nonempty) union or intersection of rings containing $S$ is also a valuation ring.

Let $u_{\mathrm{t}}$ denote the valuation on $K$ corresponding to $\mathcal{O}_{\mathbf{t}}$. We now consider a final distinction into (subsub)cases depending on the characteristic of $K u_{\mathbf{t}}$. Note that since $u_{\mathbf{t}}$ is henselian, it is a coarsening of $v_{K}$ which has mixed characteristic. Thus char $\left(K u_{\mathbf{t}}\right) \in$ $\{0, p\}$.

If, for $\mathbf{s} \in K^{n}, \phi(K, \mathbf{s})$ is a valuation ring then it will be denoted $\mathcal{O}_{\mathbf{s}}$ and its corresponding valuation will be denoted $u_{\mathrm{s}}$.

Case 2b(i): Suppose that $\operatorname{char}\left(K u_{\mathbf{t}}\right)=p$. Let

$$
\mathcal{O}_{2}:=\bigcup\left\{\phi(K, \mathbf{s}) \mid \mathcal{O}_{\mathbf{s}}=\phi(K, \mathbf{s}) \text { is a val. ring, } S \subseteq \mathcal{O}_{\mathbf{s}}, \operatorname{char}\left(K u_{\mathbf{s}}\right)=p\right\}
$$


We have the following picture.

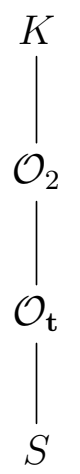

As noted above, $\mathcal{O}_{2}$ is a union of a chain of rings containing $S$, thus $\mathcal{O}_{2}$ is a valuation ring in $K$. In fact, since $\mathcal{O}_{2}$ is a union of mixed characteristic valuation rings, $\mathcal{O}_{2}$ has mixed characteristic. Thus $\mathcal{O}_{2}$ is nontrivial. By Fact 5.6, since $\mathcal{O}_{\mathbf{t}}=\phi(K, \mathbf{t}) \subseteq \mathcal{O}_{2}$, we have that $\mathcal{O}_{2}$ is henselian.

Finally, note that $\mathcal{O}_{2}$ is $\emptyset$-defined in $K$ by the following formula.

$$
\exists \mathbf{s}\left(V_{\phi}(\mathbf{s}) \wedge \forall y(y \in S \longrightarrow \phi(y, \mathbf{s})) \wedge \neg \phi\left(p^{-1}, \mathbf{s}\right) \wedge \phi(x, \mathbf{s})\right),
$$

where $V_{\phi}(\mathbf{s})$ is a formula defining those $\mathbf{s}$ such that $\phi(K, \mathbf{s})$ is a valuation ring. This finishes Case $2 \mathrm{~b}(\mathrm{i})$.

Case 2b(ii): Suppose that $\operatorname{char}\left(K u_{\mathbf{t}}\right)=0$. Let

$$
\mathcal{O}_{3}:=\bigcap\left\{\phi(K, \mathbf{s}) \mid \mathcal{O}_{\mathbf{s}}=\phi(K, \mathbf{s}) \text { is a val. ring, } S \subseteq \mathcal{O}_{\mathbf{s}}, \operatorname{char}\left(K u_{\mathbf{s}}\right)=0\right\} .
$$

We have the following picture.

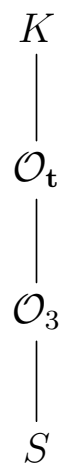

As noted above, as an intersection of a chain of rings containing $S, \mathcal{O}_{3}$ is a valuation ring in $K$. In fact, since $\mathcal{O}_{3}$ is an intersection of equal characteristic valuation rings, $\mathcal{O}_{3}$ has equal characteristic. Since $\mathcal{O}_{3} \subseteq \mathcal{O}_{\mathbf{t}}, \mathcal{O}_{3}$ is nontrivial.

We claim that $\mathcal{O}_{3}$ is a coarsening of $\mathcal{O}_{K}$, i.e. $\mathcal{O}_{K} \subseteq \mathcal{O}_{3}$. To see this: let $L \in \mathcal{L}$. As noted above, $\mathcal{O}_{L}^{q} \cap K$ is comparable to $\mathcal{O}_{K}$. Either

$$
\mathcal{O}_{K} \subseteq \mathcal{O}_{L}^{q} \cap K \subseteq S \subseteq \mathcal{O}_{3},
$$

as required; or

$$
\mathcal{O}_{L}^{q} \cap K \subset \mathcal{O}_{K}
$$

In the latter case, $\mathcal{O}_{K}$ and $\mathcal{O}_{3}$ are both coarsenings of $\mathcal{O}_{L}^{q} \cap K$; and so they are comparable, by Fact 5.6. Since $\mathcal{O}_{3}$ has residue characteristic zero, $\mathcal{O}_{K} \subset \mathcal{O}_{3}$. In either case, we have shown that $\mathcal{O}_{3}$ is a coarsening of $\mathcal{O}_{K}$. Consequently, $\mathcal{O}_{3}$ is henselian. 
Since $S$ is a union of valuation rings of residue characteristic $p$, if $\phi(K, \mathbf{s})=\mathcal{O}_{\mathbf{s}}$ is a valuation ring that contains $S$, then $\mathcal{O}_{\mathrm{s}}$ has residue characteristic 0 or $p$. Therefore $\mathcal{O}_{3}$ is $\emptyset$-defined in $K$ by the following formula.

$$
\forall \mathbf{s}\left(\left(V_{\phi}(\mathbf{s}) \wedge \forall y(y \in S \longrightarrow \phi(y, \mathbf{s})) \wedge \phi\left(p^{-1}, \mathbf{s}\right)\right) \longrightarrow \phi(x, \mathbf{s})\right),
$$

where, as above, $V_{\phi}(\mathbf{s})$ is a formula defining those $\mathbf{s}$ such that $\phi(K, \mathbf{s})$ is a valuation ring. This finishes Case $\mathbf{2 b}(\mathbf{i i})$, and hence the proof of the theorem.

5.4. The full picture in mixed-characteristic. We can now collect the facts we have proven for fields in $\mathcal{K}_{0, p}$ and assemble them to a proof of Theorem 1.1 (B):

Proof of part (B) of Theorem 1.1. We want to show that for each prime $p$, in the class $\mathcal{K}_{0, p}$ the complete picture is

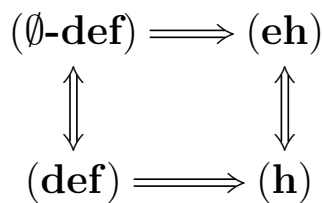

Apart from the trivial implications as given in Figure 1, we have shown in Corollary 2.2 that for any $K \in \mathcal{K}_{0, p}$

$$
(\mathbf{h}) \Longleftrightarrow(\mathbf{e h})
$$

and furthermore in Theorem 5.7 that also

$$
(\text { def }) \Longleftrightarrow(\emptyset \text {-def })
$$

holds. Finally, Example 5.5 shows that we have

$$
\text { (h) } \not(\emptyset \text {-def })
$$

in $\mathcal{K}_{0, p}$. This completes the proof.

\section{ACKNOWLEDGEMENTS}

The authors would like to extend their thanks to the Nesin Mathematics Village (https://matematikkoyu.org/eng/) for its hospitality during the research visit on which this work was begun. The authors are also very grateful to the referee for pointing out mistakes in earlier versions.

\section{REFERENCES}

[AF16] Sylvy Anscombe and Arno Fehm. The existential theory of equicharacteristic henselian valued fields. Algebra \& Number Theory, 10:665-683, 2016.

[Efr06] Ido Efrat. Valautions, Orderings, and Milnor K-Theory. American Mathematical Society, 2006.

[EP05] Antonio J. Engler and Alexander Prestel. Valued fields. Springer Monographs in Mathematics. Springer-Verlag, Berlin, 2005.

[FJ15] Arno Fehm and Franziska Jahnke. On the quantifier complexity of definable canonical henselian valuations. Mathematical Logic Quarterly, 61(4-5):347-361, 2015.

[FP11] Arno Fehm and Elad Paran. Non-ample complete valued fields. Int. Math. Res. Not. IMRN, (18):4135-4146, 2011.

[Hod97] Wilfrid Hodges. A shorter Model Theory. Cambridge University Press, Cambridge, 1997.

[Hon14] Jizhan Hong. Definable non-divisible Henselian valuations. Bull. Lond. Math. Soc., 46(1):14-18, 2014.

[JK15a] Franziska Jahnke and Jochen Koenigsmann. Defining coarsenings of valuations. Preprint, available on ArXiv:1501.04506 [math.LO], to appear in Proceedings of the Edinburgh Mathematical Society, 2015.

[JK15b] Franziska Jahnke and Jochen Koenigsmann. Uniformly defining $p$-henselian valuations. Annals of Pure and Applied Logic, 166:741-754, 2015. 
[Joh15] Will Johnson. On dp-minimal fields. Preprint, available on ArXiv:1507.02745 [math.LO], 2015.

[Kap42] Irving Kaplansky. Maximal fields with valuations. Duke Math. J., 9(2):303-321, 1942.

[Koe94] Jochen Koenigsmann. Definable valuations. Preprint, 1994.

[Kru15] Krzysztof Krupiński. Superrosy fields and valuations. Ann. Pure Appl. Logic, 166(3):342-357, 2015.

[Kuh04] Franz-Viktor Kuhlmann. Value groups, residue fields, and bad places of rational function fields. Transactions of the American Mathematical Society, 11(356):4559-4600, 2004.

[Kuh10] Franz-Viktor Kuhlmann. A classification of artin-schreier defect extensions and characterizations of defectless fields. Illinois Journal of Mathematics, 54(2):397-448, 2010.

[Kuh16] Franz-Viktor Kuhlmann. The algebra and model theory of tame valued fields. J. reine angew. Math., 719:1-43, 2016.

[Lan05] Serge Lang. Undergraduate Algebra. Springer, 2005.

[PD11] Alexander Prestel and Charles N. Delzell. Mathematical logic and model theory. Universitext. Springer, London, 2011. A brief introduction, Expanded translation of the 1986 German original.

[Pre91] Alexander Prestel. Algebraic number fields elementarily determined by their absolute galois group. Israel J. Math., 73:199-205, 1991.

[Pre15] Alexander Prestel. Definable henselian valuation rings. J. Symbolic Logic, 80:1260-1267, 2015.

[PZ78] Alexander Prestel and Martin Ziegler. Model-theoretic methods in the theory of topological fields. J. Reine Angew. Math., 299 (300):318-341, 1978.

[TZ12] Katrin Tent and Martin Ziegler. A course in model theory, volume 40 of Lecture Notes in Logic. Association for Symbolic Logic, La Jolla, CA, 2012.

Jeremiah Horrocks Institute, Leighton Building Le7, University of Central LanCAshire, Preston PR1 2HE, United Kingdom

E-mail address: sanscombe@uclan.ac.uk

Institut für Mathematische Logik und Grundlagenforschung, University of Münster, Einsteinstr. 62, 48149 Münster, GERMAnY

E-mail address: franziska.jahnke@wwu.de 